\title{
Properties of general relativistic, irrotational binary neutron stars in close quasiequilibrium orbits : Polytropic equations of state
}

\author{
Kōji Uryu $\bar{u}^{1}$, Masaru Shibata ${ }^{2,3}$ and Yoshiharu Eriguchi ${ }^{4}$ \\ ${ }^{1}$ SISSA, Via Beirut 2/4, Trieste 34013, Italy \\ ${ }^{2}$ Department of Physics, University of Illinois at Urbana-Champaign, Urbana, IL 61801, USA \\ ${ }^{3}$ Department of Earth and Space Science, Graduate School of Science, Osaka University, \\ Toyonaka, Osaka 560-0043, Japan \\ ${ }^{4}$ Department of Earth Science and Astronomy, Graduate School of Arts and Sciences, University of Tokyo, \\ Komaba, Meguro, Tokyo 153-8902, Japan
}

\begin{abstract}
We investigate close binary neutron stars in quasiequilibrium states in a general relativistic framework. The configurations are numerically computed assuming (1) existence of a helicoidal Killing vector, (2) conformal flatness for spatial components of the metric, (3) irrotational velocity field for the neutron stars and (4) masses of neutron stars to be identical. We adopt the polytropic equation of state and the computation is performed for a wide range of the polytropic index $n(=0.5,0.66667,0.8,1,1.25)$, and compactness of neutron stars $(M / R)_{\infty}(=0.03-0.3)$, where $M$ and $R$ denote the mass and radius of neutron stars in isolation. Because of the assumption of the irrotational velocity field, a sequence of fixed rest mass can be identified as an evolutionary track as a result of radiation reaction of gravitational waves. Such solution sequences are computed from distant detached to innermost orbits where a cusp (inner Lagrange point) appears at the inner edges of the stellar surface. The stability of orbital motions and the gravitational wave frequency at the innermost orbits are investigated. It is found that the innermost stable circular orbits (ISCO) appear for the case of stiff equation of state with $n \lesssim 2 / 3$. We carefully analyze the ISCO for $n=0.5$ and show that the ISCO are mainly determined by a hydrodynamic instability for $(M / R)_{\infty} \lesssim 0.2$. We also investigate the total angular momentum and the specific angular momentum distribution of the binary configuration at the innermost orbits, where the final merger process starts. From these quantities, we expect the final outcomes of the binary neutron star coalescence.
\end{abstract}

PACS number(s): 04.25.Dm, 04.30.Db, 04.40.Dg, 97.60.Jd

\section{INTRODUCTION}

The last 10 minutes of binary neutron stars is one of the most important targets of laser interferometric gravitational wave detectors such as LIGO [1]2], as well as a possible candidate for various astrophysical phenomena such as $\gamma$-ray bursts [3] and optical transient events [ [4]. The close binary neutron stars are in quasi circular orbits and evolve as a result of gravitational wave emission towards merger. The evolutionary track of binary neutron stars is roughly divided into three phases; (i) inspiraling phase in which the characteristic orbital separation $a$ is much larger than the radius of neutron stars $R$ and the binary evolves adiabatically, (ii) intermediate phase in which the binary still evolves in the adiabatic manner but $a / R$ is so small $(\sim 2-5)$ that the effects of tidal deformation of the neutron stars become important, and (iii) the final coalescing phase in which the two neutron stars merge on the dynamical timescale. The phase (i) has been treated by an analytic method using a post Newtonian approximation and equations of motion for point particles, resulting in recent successful developments [5]. On the other hand, phases (ii) and (iii) have to be treated numerically taking into account effects of the finite size, tidal deformation and hydrodynamic interaction of the two neutron stars. In this paper, we focus on phase (ii).

The signal of gravitational waves in phases (ii) and (iii) is expected to give a wide variety of information about neutron stars and strong gravitational fields. In particular, the signal around the innermost stable circular orbit (ISCO) at which the orbit of binaries becomes dynamically unstable to starting to merge can bring important information about the structure of the neutron stars. At the ISCO, the signal is likely to change abruptly from a quasi periodic wave form to a burst wave form. In the frequency domain, such a change will be recognized as a clear cliff [6]. The characteristic frequency at the edge of the cliff may be used for extracting information about the nature of equations of state of high density neutron star matter, because the location of the ISCO may depend sensitively on the equations of state (or compactness) of the neutron stars 迎. The expected frequency for the 
ISCO, $f_{\mathrm{GW}}$, can be estimated as

$$
f_{\mathrm{GW}}=\frac{\Omega}{\pi} \simeq 860 \mathrm{~Hz}\left(\frac{M_{0} \Omega}{0.02}\right)\left(\frac{M_{0}}{1.5 M_{\odot}}\right)^{-1},
$$

where $\Omega, M_{0}$ and $M_{\odot}$ are the angular velocity, the rest mass of each star and the solar mass. Thus, for a typical value of $M_{0} \sim 1.5 M_{\odot}, f_{\mathrm{GW}}$ may be smaller than $1 \mathrm{kHz}$, which will be in the sensitive frequency band of laser interferometers [7], for a relatively small value of $M_{0} \Omega \sim 0.02$ (see, e.g., Tables II-III). This implies that the characteristic frequency may be detected by laser interferometers in the near future to constrain the equation of state of neutron stars. One of the main purposes of this paper is to investigate $f_{\mathrm{GW}}$.

Other motivations for this paper are to prepare initial conditions for numerical simulations of merging of binary neutron stars in full general relativity as well as to predict possible outcomes in simulations starting from such initial data sets. Recently, several groups have been developing numerical codes for such simulation [8 12], and it is now becoming feasible to perform the simulation stably and fairly accurately (see, e.g., [11]). As indicated in [11], outcomes of the merger depend sensitively on initial velocity field and compactness of the neutron stars, so that we need to prepare the initial conditions as realistically as possible in order to obtain reliable results. It should also be emphasized that the simulation is not still an easy task and that it is very helpful to retain a reliable method to cross-check the results. Careful analysis of the initial data sets is one of the suitable methods for such cross-checking.

In this paper, we present numerical solutions for irrotational binary neutron stars of equal mass in quasiequilibrium states. We construct such states assuming the existence of a helicoidal Killing vector (see Eq. (住) because the emission timescale for gravitational waves is longer than the orbital period even just before merging and hence the system is almost in a stationary state in the comoving frame. We also assume an irrotational velocity field for the neutron stars because it is recognized as a realistic one for binary neutron stars as long as the spin period of the neutron stars is not as short as about 1 millisecond [13]. Under these assumptions, the relativistic Euler equation can be integrated to give a Bernoullitype equation 14 16], resulting in a great simplification for handling the matter equations.

We adopt a polytropic equation of state in the form

$$
P=\kappa \rho^{1+1 / n}
$$

where $P, \rho, n$ and $\kappa$ are the pressure, rest mass density, polytropic index, and polytropic constant, respectively. The adiabatic assumption (i.e., $\kappa$ is a constant) is appropriate because the timescale for heating and cooling inside neutron stars is much longer than the inspiraling timescale of binaries due to gravitational radiation. Since realistic equations of state are not clear yet [17], we choose a wide range for $n$ between $n=0.5$ and 1.25 so that various moderately stiff equations of state for neutron star matter can be approximated. We also survey a wide range of the compactness parameter from $(M / R)_{\infty}=0.03$ to 0.3 where $(M / R)_{\infty}$ denotes the compactness of neutron stars in isolation. With these parameter sets, we will be able to explore the effects of various realistic equations of state appropriately.

This paper is organized as follows : In Sec. II, we briefly review our computational method and definition of physical quantities. In Sec. III, we show numerical results. We analyze the quasiequilibrium states from various points of view. First, we construct a large number of quasiequilibrium sequences of constant rest mass for a wide range of $n$ and $(M / R)_{\infty}$. Then, we investigate the stability of orbital motions for these sequences. The stability is analyzed by searching the simultaneous minima of energy and angular momentum as a function of the orbital separation (or angular velocity) along each sequence [18, 19]. The mechanism of the instability and the gravitational wave frequency at the innermost orbits are determined. We also show that the maximum density decreases with decreasing orbital separation. Finally, we analyze the non-dimensional angular momentum parameter $q \equiv J_{\text {tot }} / M_{\mathrm{ADM}}^{2}$, where $J_{\text {tot }}$ and $M_{\mathrm{ADM}}$ are the total angular momentum and ADM mass of the system, and the distribution of the specific angular momentum of mass elements for binaries at the innermost orbits to predict possible outcomes after the merger. In Sec. IV, we summarize the results presented in Sec. III. In the Appendix, we demonstrate for completeness that neutron stars in the irrotational binary systems computed in this paper are stable against radial gravitational collapse. Throughout this paper, we take units in which $G=1=c$ where $G$ and $c$ are the gravitational constant and speed of light. Numerical computations are performed using spherical polar coordinates $(r, \theta, \varphi)$. Greek and Latin indices run over $t$ (or 0$), r, \theta, \varphi$ and $r, \theta, \varphi$, respectively. 


\section{THE METHOD FOR CONSTRUCTING A QUASIEQUILIBRIUM SEQUENCE}

\section{A. Outline of formulation}

Orbits of binary neutron stars shrink as a result of radiation reaction to gravitational waves. The ratio of the radiation reaction timescale to the orbital period is evaluated to be 20]

$$
\sim \frac{5}{128 \pi}\left(\frac{a}{M_{\mathrm{ADM}}}\right)^{5 / 2} \simeq 1.1\left(\frac{a}{6 M_{\mathrm{ADM}}}\right)^{5 / 2}
$$

In deriving Eq. (3), we adopted the quadrupole formula and the energy equation in Newtonian theory. Eq. (3) implies that even just before the merger at $a \gtrsim 6 M_{\mathrm{ADM}}$, the reaction timescale is still longer than the orbital period and that the binary is approximately in a quasi stationary state in the comoving frame with orbital angular velocity $\Omega$. Thus, neglecting the small effect of gravitational radiation reaction, we assume the existence of a helicoidal Killing vector in the form

$$
\ell^{\mu}=\left(\frac{\partial}{\partial t}\right)^{\mu}+\Omega\left(\frac{\partial}{\partial \varphi}\right)^{\mu} .
$$

Next, we assume that the velocity field of the neutron stars is irrotational. Viscosity inside neutron stars is considered to be too weak to synchronize the spin with the orbital rotation on the emission timescale for gravitational waves by the binary 13 . In the final stage of inspiraling, the orbital period is about 2 milliseconds. Thus, if the spin period of the neutron stars is much longer than 2 milliseconds, the effect of the spin just before the merger is negligible so that the irrotational velocity field can be approximately achieved. In this assumption, furthermore, the relativistic Euler equation can be integrated to give a Bernoulli-type equation [14 16. Consequently, procedure for obtaining a solution of the hydrodynamic equations is considerably simplified.

Finally, we assume that the spatial component of the metric $\gamma_{i j}$ is conformally flat and write the line element in the form 21 24

$$
d s^{2}=-\left(\alpha^{2}-\beta_{i} \beta^{i}\right) d t^{2}+2 \beta_{i} d x^{i} d t+\Psi^{4} f_{i j} d x^{i} d x^{j},
$$

where $\alpha, \beta^{i}, \Psi$ and $f_{i j}$ are the lapse function, shift vector, conformal factor, and flat spatial metric, respectively. The elliptic-type equations for $\alpha, \beta^{i}$ and $\Psi$ are derived from the Hamiltonian constraint, momentum constraint and slicing condition in which $K_{i j} \gamma^{i j}=0$ with the assumption $\gamma_{i j}=\Psi^{4} f_{i j}$ 21 24 where $K_{i j}$ denotes the extrinsic curvature. The three metric is assumed to be conformally flat for simplicity. Although the solutions obtained in this framework are valid in general relativity in the sense that they satisfy the Hamiltonian and momentum constraints, they are approximate as quasiequilibrium states because of the simplified formulation. We suspect that the solutions shown below slightly and systematically deviate from the correct ones [25]. It should always be kept in mind that we need to develop a better theoretical framework to suppress the slight deviation (see [26] for one proposal).

Solutions of quasiequilibrium states of irrotational binary neutron stars are computed using a numerical code developed by Uryū and Eriguchi [24]. The numerical method is described in 27,24 to which the reader is referred for details. The code has been tested by comparing with numerical results of other groups (e.g., 22]), as well as by carrying out several convergence tests [28].

\section{B. Definition of variables}

First, we define the total rest mass $M_{0}$, ADM mass $M_{\mathrm{ADM}}$ and angular momentum $J_{\text {tot }}$ as

$$
\begin{aligned}
M_{0, \text { tot }} & =\int \rho \alpha u^{0} \Psi^{6} d V \\
M_{\mathrm{ADM}} & =\int\left[\rho h\left(\alpha u^{0}\right)^{2}-P+\frac{1}{16 \pi} K_{i j} K^{i j}\right] \Psi^{5} d V, \\
J_{\text {tot }} & =\int \rho h \alpha u^{0} u_{\varphi} \Psi^{6} d V,
\end{aligned}
$$

where $u^{\mu}$ denotes the four velocity and $h=1+(n+1) P / \rho$. The integral is carried out over the whole three space.

We define the coordinate length of the semi-major axis $R_{0}$ and half of orbital separation $d$ as

$$
\begin{aligned}
& R_{0}=\left(R_{\text {out }}-R_{\text {in }}\right) / 2, \\
& d=\left(R_{\text {out }}+R_{\text {in }}\right) / 2,
\end{aligned}
$$

where $R_{\text {in }}$ and $R_{\text {out }}$ denote distances from the center of mass of the system to the inner and outer edges of the star respectively along the major axis. From these variables, we also define $\widehat{d}=d / R_{0}$ (note that $\widehat{d}=1$ when the surfaces come into contact and $\widehat{d} \rightarrow \infty$ for $d \rightarrow \infty)$. In the following, we adopt $\widehat{d}$ to specify a model along a quasiequilibrium sequence. 
We also define half of the separation $d_{G}$ in another way as

$$
d_{G}=\frac{1}{M_{0, \text { tot }}} \int|x| \rho \alpha u^{0} \Psi^{6} d V
$$

where $x$ denotes a coordinate along the major axis. Hereafter, we often refer to $d_{G}$ as the half of the orbital separation.

In this paper, we compute quasiequilibrium sequences fixing $\kappa$ to be constant. This treatment is appropriate for the late inspiraling stage of binary neutron stars because the timescale of heating and cooling is much longer than the inspiraling timescale of the binaries due to gravitational radiation reaction. Then, the physical units enter the problem only through the constant $\kappa$, which can be chosen arbitrarily or otherwise completely scaled out of the problem. Thus, we use non-dimensional quantities normalized by an appropriate power of $\kappa$

$$
\begin{aligned}
\bar{R}_{0} & =\kappa^{-n / 2} R_{0}, \\
\bar{d}_{G} & =\kappa^{-n / 2} d_{G}, \\
\bar{M}_{0, \mathrm{tot}} & =\kappa^{-n / 2} M_{0, \mathrm{tot}}, \\
\bar{M}_{\mathrm{ADM}} & =\kappa^{-n / 2} M_{\mathrm{ADM}}, \\
\bar{J}_{\mathrm{tot}} & =\kappa^{-n} J_{\mathrm{tot}}, \\
\bar{\rho} & =\kappa^{n} \rho .
\end{aligned}
$$

In the following, we often refer to a non-dimensional angular momentum parameter $q \equiv J_{\text {tot }} / M_{\mathrm{ADM}}^{2}$. For convenience, half of the total rest mass, ADM mass and angular momentum are also defined as $M_{0}=M_{0, \text { tot }} / 2$, $M=M_{\mathrm{ADM}} / 2$ and $J=J_{\mathrm{tot}} / 2$ with their normalized values $\bar{M}_{0}=\kappa^{-n / 2} M_{0}, \bar{M}=\kappa^{-n / 2} M$, and $\bar{J}=\kappa^{-n} J$. Strictly speaking, $M$ is not the mass of one star because the binding energy between two bodies and other interaction effects are included in it. However, the fraction is expected to be fairly small, so that we often refer to $M$ as the mass of each star.

\section{RESULTS}

\section{A. Stability of quasiequilibrium sequences of binary neutron stars}

Since the vorticity is almost exactly conserved during the evolution of binary neutron stars, a quasiequilibrium sequence of irrotational binary neutron stars of constant rest mass and with decreasing the orbital separation (or increasing $\Omega$ ) can be identified as an evolutionary sequence of binary neutron stars as a result of gravitational wave emission. We compute the sequences for a wide range of parameter space as $0.5 \leq n \leq 1.25$ and typically as $0.1 \leq(M / R)_{\infty} \leq 0.19$. (Hereafter, we use $(M / R)_{\infty}$ instead of the rest mass to specify a certain sequence.) In some cases, we extend the analysis to $(M / R)_{\infty}<0.1$ and $(M / R)_{\infty} \geq 0.2$. In this paper, we restrict our attention only to binary neutron stars of equal mass.

Each sequence is computed gradually decreasing $\widehat{d}$ from 3 to 1 . It is found that the sequences are always terminated at an innermost orbit with $\widehat{d}>1$ and $\bar{d}_{G}=\bar{d}_{R}$ at which neutron stars have cusps at the inner edges of the stellar surfaces. This property is found irrespective of $n$ and $(M / R)_{\infty}(\lesssim 0.2)$. The cusps correspond to the inner Lagrange (L1) points. Therefore, neutron stars at $\bar{d}_{G}=\bar{d}_{R}$ are subject to mass transfer from the cusps and are likely to form a dumbbell-like structure (i.e., the system will have a bridge between the two stars and will not be a "binary" any longer for $\bar{d}_{G}<\bar{d}_{R}$ ). Even at this orbit of cusps, the timescale of gravitational radiation reaction is longer than the orbital period, except for extremely compact binaries, so that such a dumbbelllike object could be still in a quasiequilibrium state and may be computed with the same strategy as used here. However, we stop the computation at $\bar{d}_{G}=\bar{d}_{R}$ since the present numerical code is suitable only for binary configurations. We show several physical quantities at this orbit in Table I.

To determine the dynamical stability of the orbit of binaries, we search for minima of $\bar{J}$ and $\bar{M}$ as functions of the orbital separation (or the angular velocity) along each sequence. Lai, Rasio, and Shapiro [18 have shown the existence of minima of $\bar{J}$ and $\bar{M}$ for an ellipsoidal model of a Newtonian irrotational binary, which indicates the onset of dynamical instability. (See Lombardi, Rasio and Shapiro [19] for an extension including a part with post Newtonian corrections.) These minima of $\bar{J}$ and $\bar{M}$ should appear simultaneously at the same separation because the following relativistic identity probably holds for irrotational sequences with constant rest mass;

$$
d M_{\mathrm{ADM}}=\Omega d J_{\mathrm{tot}} .
$$

In our numerical computation, this identity is satisfied typically to $\lesssim 10 \%$ except near to turning points where $\bar{M}$ and $\bar{J}$ are almost constant and consequently the difference between the neighborhoods is not accurately com- 
puted. Also, we checked that the minima of $\bar{J}$ and $\bar{M}$ appear simultaneously at the same separation $\bar{d}_{G}=\bar{d}_{\mathrm{dyn}}$ whenever they appear on a solution sequence [29]. In view of this, we define the location of the simultaneous minima of $\bar{J}$ and $\bar{M}$ as the ISCO.

In Fig. 目, we show $\bar{J}$ as a function of $\bar{d}_{G}$ for $n=0.5$, 0.66667 and 0.8 , and for $(M / R)_{\infty}=0.19$. (Note that we have found essentially the same behavior for $0.1 \leq$ $(M / R)_{\infty} \leq 0.19$.) For $n=0.5, \bar{J}$ and $\bar{M}$ have the minima at $\bar{d}_{G}=\bar{d}_{\mathrm{dyn}}>\bar{d}_{R}$ and so the orbits of the binary neutron stars will be dynamically unstable to merger before mass transfer sets in. For $n=0.66667$, the minima are located near $\bar{d}_{R}$ and so dynamical instability and mass transfer will set in almost together. On the other hand, for $n \geq 0.8$, minima are not found for $\bar{d}_{G} \geq \bar{d}_{R}$ and so the mass transfer will set in before the orbit becomes dynamically unstable.

Several quantities at $\bar{d}_{G}=\bar{d}_{\text {dyn }}$ for $n=0.5$ and 0.66667 are summarized in Tables II and III, respectively. As shown in Table I, $\widehat{d}$ at $\bar{d}_{G}=\bar{d}_{R}$ depends very weakly on $(M / R)_{\infty}$. In contrast, $\widehat{d}$ at $\bar{d}_{G}=\bar{d}_{\text {dyn }}$ becomes larger with increasing $(M / R)_{\infty}$, as shown in Tables II and III. For $n=0.5$, an ISCO always exists for any $(M / R)_{\infty} \leq 0.3$. For $n=0.66667$, no ISCO exists for the sequence of $(M / R)_{\infty}<0.17$, but there is one for $(M / R)_{\infty} \gtrsim 0.17$. (Note that the curves of $\bar{J}$ and $\bar{M}$ as functions of $\bar{d}_{G}$ suggest that $\bar{d}_{R}$ is close to $\bar{d}_{G}$ even for $(M / R)_{\infty}<0.17$ for $n=0.66667$.) The results for the smaller compactness $(M / R)_{\infty}<0.17$ with various $n$ are consistent with the result in the Newtonian limit $(M / R)_{\infty} \rightarrow 0$ [27], implying that the above property of the dynamical instability holds irrespective of compactness as long as $(M / R)_{\infty}<0.17$.

To summarize, we show a schematic figure in Fig. 2. We have found two cases for evolution of close binary neutron stars with irrotational velocity field. In one case, the sequence is terminated when cusps appear before the binaries reach the minima of $\bar{J}$ and $\bar{M}$ as shown in Fig. 22(a). In this case, mass transfer will occur before the orbit becomes dynamically unstable. Binary neutron stars with $n \gtrsim 2 / 3$ are in this category. In the other case, the binaries reach the minima before the cusps appear as shown in Fig. 2(b). In this case, the orbit becomes dynamically unstable before mass transfer sets in. Binary neutron stars with $n \lesssim 2 / 3$ are in this category. Based on this result, we hereafter refer to the orbit at $\bar{d}_{G}=\bar{d}_{\text {dyn }}$ for $n \lesssim 2 / 3$ and at $\bar{d}_{G}=\bar{d}_{R}$ for $n \geq 0.8$ as "innermost orbit".

\section{B. Frequencies of gravitational waves at the final orbit}

As discussed in Sec. I, it is important to clarify the frequency of gravitational waves at the ISCO from the viewpoint of gravitational-wave-astronomy. For $n=0.5$ and 0.66667, we find that an ISCO (i.e., a point at which dynamical instability sets in) does exist, and the frequency is computed from Tables II and III as

$$
\simeq \begin{cases}0.8\left(\frac{1.5 M_{\odot}}{M_{0}}\right) \mathrm{kHz} & \text { for }(M / R)_{\infty}=0.14 \\ 1.1-1.15\left(\frac{1.5 M_{\odot}}{M_{0}}\right) \mathrm{kHz} & \text { for }(M / R)_{\infty}=0.17 \\ 1.35-1.4\left(\frac{1.5 M_{\odot}}{M_{0}}\right) \mathrm{kHz} & \text { for }(M / R)_{\infty}=0.19 .\end{cases}
$$

We note that the ISCO here for $(M / R)_{\infty} \lesssim 0.2$ is probably determined by the hydrodynamic instability [18], but not by the general relativistic orbital instability. The reason is that the general relativistic orbital instability should not depend on $(M / R)_{\infty}$ in the absence of hydrodynamic effects [30]. Even in the presence of such effects, $M_{\mathrm{ADM}} \Omega$ should depend only weakly on $(M / R)_{\infty}$ when the general relativistic orbital instability dominates to determine the ISCO. However, for $(M / R)_{\infty} \lesssim 0.2$, $M_{\mathrm{ADM}} \Omega$ at the ISCO does depend strongly and systematically on $(M / R)_{\infty}$ (cf, Tables II and III, and Fig. 3). We will discuss this point in detail in Sec. III G.

For a realistic neutron star with $M_{0} \simeq 1.5 M_{\odot}$, the radius will be in the range between 10 and $15 \mathrm{~km}$. This implies that $(M / R)_{\infty}$ is between $0.14 \lesssim(M / R)_{\infty} \lesssim 0.2$ for a typical ADM mass $M_{\infty} \sim 1.4 M_{\odot}$ where $M_{\infty}$ denotes the ADM mass in isolation. The present result suggests that if the neutron star radius is fairly large, i.e., $\sim 15 \mathrm{~km}$, the frequency of gravitational waves at the ISCO will be less than $1 \mathrm{kHz}$ which is in the sensitive frequency band of the laser interferometers. However, if the radius of neutron stars is $\sim 10 \mathrm{~km}$, the frequency at the ISCO is larger than $1 \mathrm{kHz}$ and it will be difficult to detect gravitational waves emitted from binary neutron stars with $M_{\infty} \sim 1.4 M_{\odot}$ orbiting near the ISCO by means of the interferometers [7].

For $n=0.8$ and 1, the frequency of gravitational waves at $\bar{d}_{G}=\bar{d}_{R}$ is given approximately by Eq. 19, , implying that $f_{\mathrm{GW}}$ would be larger than the frequency shown in Eq. (19) for these cases. Hence, even for $R \sim 15 \mathrm{~km}$ and 
$M_{0} \sim 1.5 M_{\odot}$, the frequency at ISCOs may be larger than $1 \mathrm{kHz}$.

It should be pointed out that for $n \geq 0.8$, mass transfer is likely to set in before the binaries reach the ISCO as discussed above. If the timescale of mass transfer is longer than the timescale for evolution due to gravitational wave emission, the signal of the gravitational waves may not change abruptly from a quasi periodic type to a different one at this orbit. However, if the mass transfer proceeds quickly, a characteristic signal may be produced. As mentioned above, the frequency of gravitational waves at the onset of mass transfer is approximately given by Eq. (19) for $n=0.8$ and 1 . Thus, for the case of relatively soft equations of state, the characteristic signal at mass transfer may be detected for binary neutron stars of $(M / R)_{\infty} \sim 0.14$ and $M_{\infty} \sim 1.4 M_{\odot}$.

\section{Origin of the ISCO}

In Fig. 3, we plot $M_{\mathrm{ADM}} \Omega$ at ISCOs for $n=0.5$ as a function of $(M / R)_{\infty}$ using data tabulated in Table II. As shown in Fig. 3 and as argued in Sec. III B, $M_{\mathrm{ADM}} \Omega$ at the ISCOs increases with increasing $(M / R)_{\infty}$. This strongly indicates that the ISCO is determined by the hydrodynamic instability 18]. On the other hand, in the limit $(M / R)_{\infty} \rightarrow 1 / 2$, hydrodynamic effects should become less important so that the general relativistic orbital instability can determine the ISCO. In our present formulation, not only hydrodynamic but also general relativistic effects (in the framework of the conformal flatness approximation) are taken into account, which enables us to investigate the origin of the ISCO. In this subsection, we carry out a detailed analysis using the results of $n=0.5$.

As a first step, we derive fitting formulae for $(M / R)_{\infty}$ $<0.2$ to clarify the behavior of $M_{0} \Omega$ and $M \Omega$ at ISCOs as functions of $(M / R)_{\infty}$. In the derivation, the following two points are taken into account: (1) In the Newtonian limit $(M / R)_{\infty} \rightarrow 0, M_{0} \Omega(M / R)_{\infty}^{-3 / 2}$ and $M \Omega(M / R)_{\infty}^{-3 / 2}$ converge to an identical constant because they are non-dimensional and should be identical in Newtonian gravity. (2) In the Newtonian limit, the ISCO is determined by the hydrodynamic effect [18], and this is the case for the finite $(M / R)_{\infty}$ as long as $(M / R)_{\infty}$ is small. Consequently, all of the general relativistic corrections should appear in the power series of $(M / R)_{\infty}$ from the viewpoint of the post Newtonian approximation. From (1) and (2), we fix the functions of $M_{0} \Omega$ and $M \Omega$ in the form

$$
\begin{aligned}
& M_{0} \Omega(M / R)_{\infty}^{-3 / 2}=a+b(M / R)_{\infty}+c(M / R)_{\infty}^{2} \\
& M \Omega(M / R)_{\infty}^{-3 / 2}=a^{\prime}+b^{\prime}(M / R)_{\infty}+c^{\prime}(M / R)_{\infty}^{2}
\end{aligned}
$$

(namely, we expand them up to second post Newtonian order) and determine the coefficients $(a, b, c)$ and $\left(a^{\prime}, b^{\prime}, c^{\prime}\right)$ by the least square fitting. We here note that although we know the value for $a=a^{\prime}$ from the Newtonian computation [27, we do not use the data for the fitting. Instead, we compute the value at $(M / R)_{\infty}=0$ from the fitting formula and compare with the Newtonian results for a cross-checking.

In the least squares fitting, we use the data sets for $n=$ 0.5 of $0.03 \leq(M / R)_{\infty} \leq 0.17$ (or 0.19 ) shown in Tables II. The resulting coefficients for the fitting formulae are tabulated in Table IV. We may roughly conclude,

$$
\begin{gathered}
a=0.286 \pm 0.001, \quad b=0.40 \pm 0.03 \\
a^{\prime}=0.286 \pm 0.001, \quad b^{\prime}=0.22 \pm 0.02
\end{gathered}
$$

For $c$ and $c^{\prime}$, on the other hand, a large uncertainty of $O(0.1)$ exists. In a Newtonian analysis, $M_{0} \Omega(M / R)_{\infty}^{-1.5}$ $=M \Omega(M / R)_{\infty}^{-1.5} \simeq 0.284$ for $n=0.5$ [27, which agrees well with $a$ and $a^{\prime}$ in the above fitting formulae. This shows the validity of the fitting method adopted here.

In Fig. 1, $M_{0} \Omega(M / R)_{\infty}^{-1.5}$ and $M_{\mathrm{ADM}} \Omega(M / R)_{\infty}^{-1.5}$ at the ISCO are plotted as a function of $(M / R)_{\infty}$ together with results from the fitting formulae derived above. The filled circles and triangles denote the numerical results, and the solid and dotted lines denote results obtained from the fitting formulae. These figures indicate that the fitting formulae agree fairly well with numerical results for $(M / R)_{\infty} \lesssim 0.2$, implying that the post Newtonian expansion is reasonable in this regime. In particular, the fitting is very good around $(M / R)_{\infty} \lesssim 0.15$. In this regime, $M_{0} \Omega$ and $M_{\mathrm{ADM}} \Omega$ are well approximated by only the first post Newtonian correction, i.e., the $(M / R)_{\infty}^{2}$ term is not very important.

The results of the fitting imply that the frequency of the ISCO depends strongly and systematically on $(M / R)_{\infty}$. Taking into account that the ISCO in the Newtonian limit is determined by the hydrodynamic instability, we may naturally infer that the ISCO for small $(M / R)_{\infty} \lesssim 0.2$ is still determined by the hydrodynamic instability with general relativistic corrections. As we 
mentioned in Sec. IIIB, the compactness of a realistic neutron star is likely to be in the range between $0.14 \lesssim(M / R)_{\infty} \lesssim 0.2$. Therefore, the ISCO of the realistic binary neutron stars is determined by the hydrodynamic instability but not by the general relativistic instability.

For $0.2<(M / R)_{\infty} \leq 0.3$, on the other hand, the fitting formulae do not agree very well with the numerical results, implying that the angular velocity at the ISCOs seems to be affected by strong nonlinear effects of general relativity. In this regime, the hydrodynamic effects seem to be less important. In Fig. 0 , we show the ratio of semidiameters for neutron stars at the ISCOs as a function $(M / R)_{\infty}$ for $n=0.5$. The semi-diameters $\left(a_{1}, a_{2}, a_{3}\right)$ are computed from

$$
a_{n}=\int \Psi^{2} d \ell \quad(n=1,2,3),
$$

where the line integral is taken along straight lines in Cartesian coordinates, and the three directions $(n=$ $1-3)$ are chosen to be orthogonal each other. $a_{1}$ is the longest diameter along a line connecting the centers of the two neutron stars. $a_{2}$ and $a_{3}$ are those in the equatorial and the meridional plane, respectively, and they intersect each other at the coordinate center of $a_{1}$. The circles and crosses in Fig. 0 denote $a_{2} / a_{1}$ and $a_{3} / a_{1}$, respectively. These ratios appear to converge towards unity with increasing $(M / R)_{\infty}$. This indicates that the tidal effect is less important for determination of the ISCO for $(M / R)_{\infty} \sim 0.3$. However, even in this highly general relativistic regime, $M_{\mathrm{ADM}} \Omega$ still does not converge to a constant as shown in Fig. 3. Since $M_{\mathrm{ADM}} \Omega$ depends strongly on $(M / R)_{\infty}$, the effect of the hydrodynamic instability still appears to dominate over the general relativistic orbital instability for determining the ISCO.

Cook [31] and recently Baumgarte [32] have investigated the ISCO for binary black holes using the conformal flatness approximation for the geometry as we do. According to their results, $\mathcal{M} \Omega$ at the ISCO is $0.17-$ 0.18. ( $\mathcal{M}$ is the sum of the gravitational masses of the two black holes, and is slightly different from $M_{\mathrm{ADM}}$ although the difference is not important here.) As shown in Fig. 3, $M_{\mathrm{ADM}} \Omega \simeq 0.11$ at the ISCO for $(M / R)_{\infty}=0.3$ and it is still monotonically increasing. This tendency does not contradict their results. The ISCO in their work is purely determined by the general relativistic orbital instability. Therefore, we may expect that the
ISCO of binary neutron stars is eventually determined by the general relativistic orbital instability for a sufficiently large $(M / R)_{\infty}>0.3$ and $M_{\mathrm{ADM}} \Omega$ converges to a constant $\sim 0.17-0.18$, although such a large compactness is unrealistic for neutron stars. (We have the value $M_{\mathrm{ADM}} \Omega \simeq 0.18$ for $n=0.5$ case, if we naively extrapolate data points at the ISCO with $(M / R)_{\infty}=0.24$ and 0.3 linearly to $(M / R)_{\infty}=4 / 9$.)

Finally, we compare the present results with ones computed using other analytic methods. Kidder, Will and Wiseman [33], Damour, Iyer and Sathyaprakash [34], and Buonanno and Damour 35] have determined the ISCO using relativistic equations of motion for two point particles in which some general relativistic corrections have been taken into account. They have derived the orbital angular velocity at the ISCO as $\mathcal{M} \Omega \simeq 0.0605$, 0.08850 and 0.07340 , respectively. Since equations of motion for point particles have been used in their methods, the ISCO has been determined by the general relativistic orbital instability and $\mathcal{M} \Omega$ has been independent of $(M / R)_{\infty}$. This is in contrast with our results, in which $M_{\mathrm{ADM}} \Omega$ depends on $(M / R)_{\infty}$ even around $(M / R)_{\infty} \sim 0.3$ and consequently the general relativistic orbital instability does not seem to dominate.

The reason for this contradiction is clear for $(M / R)_{\infty}$ $\lesssim 0.2$. As we showed above, the ISCO for $(M / R)_{\infty} \lesssim 0.2$ is determined by the hydrodynamic instability. In this regime for the compactness, $M_{\mathrm{ADM}} \Omega \lesssim 0.06$ which is smaller than their results, namely, that before the general relativistic effects between two stars become important, the orbits become unstable because of the hydrodynamic instability. Since realistic neutron stars have $0.14 \lesssim(M / R)_{\infty} \lesssim 0.2$, the approaches using equations of motion for point particles are not appropriate for investigating the ISCO of binary neutron stars.

For $0.2<(M / R)_{\infty} \leq 0.3$ and $M_{\mathrm{ADM}} \Omega \leq 0.11$, our present results suggest that the ISCO may be determined by the hydrodynamic instability. According to the point particle approaches, however, the general relativistic orbital instability should be important for $M_{\mathrm{ADM}} \Omega>0.06-0.09$ implying that our results contradict theirs in this regime, too. The reason for this contradiction is not clear. The works of ourselves, Cook and Baumgarte are based on the conformal flatness approximation which may fail to include some important general relativistic effects [25]. The neglected effects might play an important role in determining the ISCO for the regime 
$(M / R)_{\infty}>0.2$. Thus, we should not draw any strong conclusions for such highly relativistic binaries from results obtained in this framework since this might be the reason for the contradiction. To pin down the uncertainty for the high compactness regime, we need to carry out computations including higher general relativistic corrections.

\section{Evolution of the maximum density}

In Figs. 6(a)-(c), we show the relative change in the maximum energy density of a star $\Delta e \equiv\left(e_{\max }-e_{\infty}\right) / e_{\infty}$ as a function of $\widehat{d}$ for $n=0.5,0.66667$ and 0.8 , respectively. Here, $e=\rho+n P$, and $e_{\max }$ and $e_{\infty}$ denote respectively its maximum value for $\widehat{d}<\infty$ and its value for an isolated spherical star $(\widehat{d}=\infty)$ as computed from the TOV equation.

The figures indicate that the maximum density slightly decreases with decrease of the orbital separation irrespective of $n$ for $\widehat{d} \lesssim 2$. For larger separations of $\widehat{d} \gtrsim 2$, each star becomes almost spherical so that $\Delta e$ can be less than the numerical uncertainty. The small global shifts $(<1 \%)$ of each curve from zero for $2 \lesssim \widehat{d} \lesssim 3$ are thought to be due to numerical errors associated with our numerical scheme (a finite difference scheme). Small deviations around the average for $2 \lesssim \widehat{d} \lesssim 3$ are due to a systematic error associated with the Legendre expansion for the gravitational field. (See 24] for details.) Thus, our computation indicates no evidence that neutron stars in binary systems become dynamically unstable against gravitational collapse. Instead, the maximum density slightly decreases with decreasing orbital separation irrespective of $n$. (See the Appendix for more detailed computations for selected values of $n$.) This result is consistent with recent analytic calculations in general relativity 36.

\section{E. Prospects for the outcome after the merger}

First, we investigate the quantity $q \equiv J_{\text {tot }} / M_{\mathrm{ADM}}^{2}$ which is an important parameter for predicting the outcome after a merger. If the $q$ of the object resulting from the merger is larger than unity, it will not be able to collapse to form a black hole. Thus, if $q$ is larger than unity for a binary at its innermost orbit, there is a possibility that no black hole will be formed promptly after the merger. On the other hand, if $q$ is less than unity before the merger, we could consider that the system is a candidate to become a black hole, because $q$ will not increase during the merger due to emission of gravitational waves 37.

In Fig. 17, we show the relation between $(M / R)_{\infty}$ and $q$ at the innermost orbits of irrotational binary neutron stars for $n=0.5-1.25$ and we also show the relation for corotating binary neutron stars with $n=1$ for comparison. Here, the innermost orbits for irrotational binaries are the orbits at $\bar{d}_{G}=\bar{d}_{\text {dyn }}$ for $n=0.5$ and at $\bar{d}_{G}=\bar{d}_{R}$ for $n \geq 2 / 3$ as we define in Sec. IIIA. (Note that $\bar{d}_{R} \simeq \bar{d}_{\text {dyn }}$ for $n=2 / 3$.) For corotating binaries, we choose the orbit at the energy and angular momentum minima. It is found that $q$ is always less than unity irrespective of $n$ for irrotational binaries with $(M / R)_{\infty} \geq 0.13$. As mentioned in Sec. IIIA, $(M / R)_{\infty}$ will be larger than $\sim 0.14$ for a realistic neutron star. Therefore, $q$ for realistic binary neutron stars decreases below unity before merger, implying that the binary system satisfies a necessary condition to form a black hole.

Here, two cautions are appropriate. (1) For very stiff equations of state, the total rest mass of the system can be smaller than the maximum allowed rest mass of a spherical star. Indeed, for $n=0.5$ and $(M / R)_{\infty}=0.14$, this is the case. Thus, for very stiff equations of state, a black hole will not be formed even if $q<1$ before merger and $(M / R)_{\infty}$ is fairly large $(\sim 0.14)$. (2) Even in the case when the system is supramassive in the sense that the rest mass of the system is larger than the maximum rest mass of a spherical star with the same equation of state, the condition $q<1$ is not sufficient for black hole formation, because rapid rotation could support the self-gravity of the supramassive star [38]. To find the outcome of the merger, numerical simulations are obviously necessary.

In contrast with the above results, $q$ is larger than unity even for $(M / R)_{\infty} \sim 0.17$ at the energy and angular momentum minima for corotating binary neutron stars with $n=1$ [39]. The reason for the difference between the two cases is that stars in the irrotational binaries have only negligible spin angular momentum while those in the corotating binaries have significant spin angular momentum. In the study of corotating binary neutron stars with $(M / R)_{\infty} \sim 0.17$, one could reach the conclusion that a black hole might not be formed after the merger, which is completely different from our present conclusion for irrotational binary neutron stars. This result brings us to another caution, that if we adopted corotating velocity 
fields, we could reach an incorrect conclusion for realistic binary neutron stars which will have (nearly) irrotational velocity fields.

Next, we investigate the mass spectrum with respect to the specific angular momentum of fluid elements in order to estimate the mass of the disk around a black hole formed after merger. We consider this for binaries at innermost orbits which are chosen in the same manner as above. The mass spectrum with respect to the specific angular momentum is defined as

$$
\frac{M_{0}(j)}{M_{0}}=\frac{1}{M_{0}} \int_{j^{\prime}>j} \rho \alpha u^{0} \Psi^{6} d V
$$

where the integral is performed for fluid elements which have specific angular momentum larger than a given value $j$. The specific angular momentum $j$ is defined as

$$
j=h u_{\varphi} .
$$

Here, we note that $j$ of each fluid element is exactly conserved in axisymmetric systems for ideal fluid. For nonaxisymmetric systems, it may not be conserved due to some dissipation processes such as gravitational radiation or other outward transportation process.

In Figs. 8, we show $M_{0}(j) / M_{0}$ as a function of $j / M_{\mathrm{ADM}}$ for $n=0.5-1.25$, and $(M / R)_{\infty}=0.14$ and 0.17 . Here, we choose binaries with $(M / R)_{\infty} \geq 0.14$ in order to be realistic. The vertical dotted lines in Figs. 8 denote $j / M_{\mathrm{ADM}}$ of a test particle orbiting at the ISCO around a Kerr black hole for $q=0.99,0.95$, and 0.9 [20], showing the minimum allowed values for test particles orbiting a black hole. For $n=1$ (Fig. $8(\mathrm{~d})$ ), we also show the results for corotating binaries for comparison. The figures show that the mass fraction of fluid elements with $j / M_{\mathrm{ADM}}>1.6$ for irrotational binary neutron stars is zero irrespective of $n$ and $(M / R)_{\infty}$. In contrast, that for corotating binaries is $\sim 5 \%$ even for $j / M_{\mathrm{ADM}}=2$.

Now, we assume the following hypotheses which are likely to hold in the merger of binary neutron stars with $(M / R)_{\infty} \geq 0.14$ : (1) A black hole is formed. This assumption could be wrong for $n=0.5$ and $(M / R)_{\infty} \sim$ 0.14 , but would be correct for a moderate stiffness, $2 / 3 \lesssim n \lesssim 1$ (for $n=1$, numerical simulations have indicated the correctness of this assumption [11]). (2) The disk mass formed after the merger is much smaller than the black hole mass (say at most $10 \%$ of the total rest mass), and consequently the mass of black hole $M_{\mathrm{BH}}$ is nearly equal to the initial gravitational mass $M_{\mathrm{ADM}}$ (say $\left.\geq 0.9 M_{\mathrm{ADM}}\right)$. (3) The specific angular momentum $j$ for most of fluid elements changes by at most $10 \%$ during the merger process. One mechanism to change $j$ is gravitational radiation which would decrease $j$ of all fluid elements by $\lesssim 10 \%$. The other is hydrodynamic interaction which could transport $j$ outward and consequently a part of fluid elements could have larger $j$ than its original value. Therefore this hypothesis would be reasonable if the merger process to form the black hole proceeds on the dynamical timescale of the system and it is shorter than the timescale for the outward transportation of angular momentum by the hydrodynamic interaction 40]. (4) The quantity $q$ of the black hole finally formed is about $10 \%$ smaller than the initial value for the system because of gravitational radiation reaction [37] (i.e., $q \lesssim 0.9$ ).

With the hypotheses (1), (2) and (4), the specific angular momentum of a test particle at the ISCO around the formed black hole is approximately determined from the $M_{\mathrm{ADM}}$ and $J_{\text {tot }}$ given initially. As shown above, $q$ for binaries with $(M / R)_{\infty} \geq 0.14$ is less than unity and according to (4), $q$ of the black hole will be $\lesssim 0.9$ after the merger. This implies that the specific angular momentum $j$ at the ISCO around the black hole would be larger than $2 M_{\mathrm{BH}}$ [41], and to form disks, there have to exist a fraction of fluid elements of $j \gtrsim 2 M_{\mathrm{BH}} \gtrsim 1.8 M_{\mathrm{ADM}}$. However, as shown in Figs. 8, the mass of the fluid element of $j / M_{\mathrm{ADM}}>1.6$ is almost zero before merger for any $n$. Thus, if all the hypotheses are correct, the mass fraction of the disk around the black hole is almost zero after the merger of irrotational binary neutron stars of $(M / R)_{\infty} \geq 0.14$. In contrast, the mass fraction of the disk with $j \gtrsim 2 M_{\mathrm{BH}}$ can be about $\sim 5 \%$ for corotating case as shown in Fig. B(d).

Recent numerical simulations of merging binary neutron stars of equal mass in full general relativity for $n=1$ [11] indicate that for irrotational binaries, the mass of the disk around the black hole formed after merger is negligible, while for corotating binaries, it could be $\sim 5-10 \%$. These results are in complete agreement with the above analysis. This suggests that the above working hypotheses are approximately correct. Also, it suggests that the present analysis can be applied for other $n$. Thus, irrespective of $n$ and $(M / R)_{\infty}(\geq 0.14)$, a massive disk is unlikely to be formed around the black hole after mergers of irrotational binary neutron stars.

The present result leads us to give a strong caution 
with regard to previous numerical studies. As shown above, the fraction of the disk mass depends sensitively on the initial velocity field and effect of general relativity (which determines the minimum allowed value of the specific angular momentum of the disk). In the past decade, most of simulations of binary neutron stars of equal mass have been performed using rather crude initial conditions for the velocity field and without general relativistic effects (see, e.g., 42 for review). Such simulations in some cases have provided results in which a disk is formed around the merged objects. However, the present analysis suggests that such results might be wrong and gives a warning that it is dangerous to draw any conclusion about the mass of the disk without fully including general relativistic effects and without using correct velocity fields for the initial conditions.

\section{SUMMARY}

We have presented numerical results for quasiequilibrium sequences of irrotational binary neutron stars of equal mass in general relativity adopting the polytropic equation of state $p=\kappa \rho^{1+1 / n}$. Computations have been performed for a wide range of the polytropic index $n$ and the compactness $(M / R)_{\infty}$. We have analyzed the sequences from various points of view. The conclusions obtained in this paper are as follows.

The dynamical instability for orbits of irrotational binary neutron stars sets in before mass transfer occurs for $n \lesssim 2 / 3(\Gamma \gtrsim 2.5)$. Thus, the binary reaches the ISCO without mass transfer in this case. On the other hand, for $n \gtrsim 2 / 3(\Gamma \lesssim 2.5)$, mass transfer will occur before binaries reach the ISCO. This property depends very weakly on the compactness for $(M / R)_{\infty} \lesssim 0.17$. The frequency of gravitational waves at the innermost orbit (ISCO or the point where mass transfer sets in) is about 800 to 1500 $\mathrm{Hz}$ depending on the compactness. For a binary of less massive (i.e., less compact) neutron stars, the frequency could be less than $1000 \mathrm{~Hz}$ and as a result the characteristic signal of gravitational waves emitted around the ISCO may be detected by laser interferometric detectors.

We have determined the ISCOs for a wide range of $(M / R)_{\infty} \leq 0.3$ for $n=0.5$ and of $0.17 \leq(M / R)_{\infty} \leq$ 0.27 for $n=0.66667$. The results indicate that the ISCO is determined by the hydrodynamic instability and not by the general relativistic orbital instability for realistic binary neutron stars with $0.14 \lesssim(M / R)_{\infty} \lesssim 0.2$. Our present results indicate that the approaches using equations of motion for point particles are not appropriate for determining the ISCO of binary neutron stars if the neutron stars are not extremely compact $(M / R)_{\infty} \gg 0.2$.

The maximum density of neutron stars in irrotational binary systems decreases as the orbital separation decreases. Associated with this, we show that neutron stars in irrotational binary systems (which are stable in isolation) are stable against gravitational collapse until the merger sets in (see Appendix for details).

The angular momentum parameter $q=J_{\text {tot }} / M_{\text {ADM }}^{2}$ is less than unity for $(M / R)_{\infty} \gtrsim 0.13$ irrespective of $n$ for irrotational binaries at the innermost orbits (i.e. $\bar{d}_{G}=\bar{d}_{R}$ or $\left.\bar{d}_{\mathrm{dyn}}\right)$. Since $(M / R)_{\infty}$ of realistic neutron stars is expected to be larger than 0.14 , the result implies that realistic binary neutron stars satisfy a necessary condition for forming black hole, $q<1$, before the merger. However, this condition is not sufficient for determining the outcome after the merger as mentioned in Sec. IIIE. Numerical simulations in full general relativity are obviously necessary to clarify the nature of the object resulting from the merger [11].

It is found that the specific angular momentum of all of the fluid elements in irrotational binary neutron stars of equal mass will become very small $\left(j / M_{\mathrm{ADM}}<1.6\right)$ before merger irrespective of $n$ and $(M / R)_{\infty}$. As long as outward transportation of angular momentum does not work effectively during merging, the disk mass around a black hole which would be formed after the merger for $(M / R)_{\infty} \gtrsim 0.14$ will be negligible. We should note here that the present conclusion is obtained for binaries of equal mass (or nearly equal mass). In the case when the mass ratio of two stars deviates from unity by a large factor, the star of smaller mass could be tidally disrupted by the companion of larger mass at a fairly large orbital separation at which the specific angular momentum of fluid elements could be large enough to form a disk during the tidal disruption. For exploring the possibility for formation of a disk in this way, it is necessary to perform computations for binary neutron stars of unequal mass, and this is one of the key issues for the future work. 


\section{ACKNOWLEDGMENTS}

We would like to thank Prof. J. C. Miller for carefully reading of the manuscript, helpful comments and continuous encouragement. We also would like to thank Dr. M. Ruffert for useful discussions and comments on the manuscript. K.U. would like to thank Prof. D. W. Sciama for his warm hospitality at SISSA and ICTP. M.S. thanks JSPS and the Department of Physics, University of Illinois for hospitality. Numerical computations were in part carried out at the Astronomical Data Analysis Center of the National Astronomical Observatory of Japan.

\section{APPENDIX: STABILITY OF NEUTRON STARS AGAINST GRAVITATIONAL COLLAPSE AND EXISTENCE OF SUPRAMASSIVE IRROTATIONAL BINARY NEUTRON STARS}

It is important to check that neutron stars which are stable in isolation are stable against gravitational collapse to black holes even in binary systems. (See [21,23] about a claim that the neutron stars in irrotational binary systems may be unstable to collapse individually.) In this Appendix, we show that no evidence is found in our numerical experiment for selected values of $n$.

The concern about gravitational collapse is relevant for neutron stars of near to the maximum allowed rest mass. Thus we here focus only on such massive neutron stars. As noted in the caption of Table I, the compactness $(M / R)_{\infty}$ of neutron stars at the maximum allowed rest mass is larger than 0.25 for $n \leq 0.8, \sim 0.21$ for $n=1$ and $\sim 0.17$ for $n=1.25$. The compactness of realistic neutron stars would be in a range between $0.14 \lesssim(M / R)_{\infty} \lesssim$ 0.2 and probably less than 0.25 . From this fact, we pay attention only to $n=1$ and 1.25 here.

First, for calibrating our numerical code, we compare numerical results at large $\widehat{d}$ with those for spherical stars computed by solving the TOV equations. Then, we show the existence of supramassive neutron stars in close binary systems confirming the excess of the maximum rest mass beyond the maximum rest mass of the spherical stars. Finally, we discuss the stability against collapse of supramassive neutron stars in close binary systems (stars whose rest mass is larger than the spherical maximum mass).
In the analysis, we computed quasiequilibrium states with fixed $\widehat{d}$, changing $(M / R)_{\infty}$ (or $\left.\bar{\rho}_{\max }\right)$. In Fig. 9, we show $\bar{M}_{0}$ as a function of $\bar{\rho}_{\text {max }}$ (solid lines) (a) for $n=1$ and (b) for $n=1.25$. Here, $\widehat{d}$ is chosen between 1.3125 and 2.0 for $n=1$ and between 1.3125 and 2.5 for $n=1.25$. For obtaining the configuration at $\widehat{d}=\infty$ (denoted by the dashed lines), the TOV equations are solved. Note that the maximum mass for spherical stars is $\bar{M}_{0} \simeq 0.180$ at $\bar{\rho}_{\max } \simeq 0.318\left((M / R)_{\infty} \simeq 0.214\right)$ for $n=1$ and $\bar{M}_{0} \simeq 0.2167$ at $\bar{\rho}_{\max } \simeq 0.148\left((M / R)_{\infty} \simeq\right.$ 0.172 ) for $n=1.25$.

For large separation $\widehat{d} \gtrsim 2.0$, neutron stars in irrotational binary systems become almost spherical irrespective of $n$. Accordingly, the solid curves should converge toward the dashed curve with increasing $\widehat{d}$. Figure 9 shows that this is approximately the case, but the curve in the limit $\widehat{d} \gg 1.0$ slightly deviates from the dashed curve. This deviation is due to a systematic error in numerical computation. This shows that the present numerical code overestimates the rest mass by $\sim 0.4 \%$ for a quasiequilibrium state with a given $\bar{\rho}_{\text {max }}$. For this reason, the curve in the limit $\widehat{d} \gg 1.0$ denotes the relation between the rest mass and $\bar{\rho}_{\max }$ for spherical stars computed in our numerical code, and hereafter, we refer to this curve as the relation for spherical stars. The maximum of this curve is $\bar{M}_{0}^{\text {smax }}=0.1802$ for $n=1$ and $=0.2174$ for $n=1.25$.

With decreasing $\widehat{d}$, the maximum value of $\bar{M}_{0}\left(\bar{\rho}_{\text {max }}\right)$ increases. This suggests that the maximum allowed rest mass of neutron stars in irrotational binary systems is increased due to the tidal effect. The maximum at $\widehat{d}=1.3125$ is $\bar{M}_{0} \simeq 0.1808$ for $n=1$ and 0.2179 for $n=1.25$. This implies that supramassive neutron stars exist in irrotational binary systems, but the maximum mass $\bar{M}_{0}$ can be increased by at most $\sim 0.3 \%$ even at $\bar{d}_{G} \simeq \bar{d}_{R}$. This increase is much smaller than that in the corotating binaries [39], for which $\bar{M}_{0}$ increases by $\sim 2 \%$ for $n=1$ and and by $\sim 1.5 \%$ for $n=1.5$. The reason is that neutron stars in irrotational binary systems have negligible spin, while those in corotating binary systems have significant spin and the centrifugal force by this can increase the maximum allowed rest mass. Such binary systems with supramassive neutron stars could be formed in principle after a supramassive core collapse to undergo bifurcation.

Baumgarte et al. [39] have discussed the stability of a supramassive neutron star in a corotating binary us- 
ing the turning point method [43. They have concluded that the supramassive and all other neutron stars below a critical density in a corotating binary are stable against gravitational collapse. Following their discussion, we plot in Fig. 10 sequences of constant $\bar{M}_{0}$ for a variety of $\bar{M}_{0}$ on the $\bar{\rho}_{\max }-\bar{M}$ plane (the solid and thick long dashed lines). Each solid curve is drawn for $1.3125 \leq \widehat{d} \leq 2.0$ in the case $n=1$ and for $1.3125 \leq \widehat{d} \leq 1.875$ in the case $n=1.25$. Here, each sequence of constant $\bar{M}_{0}$ can be interpreted as an evolutionary sequence of a binary system as discussed in section III. When a binary system evolves as a result of gravitational radiation, the gravitational mass decreases. Thus, if $\bar{\rho}_{\max }$ decreases (increases) with decreasing gravitational mass, the neutron stars are expected to be in a stable (unstable) branch. Therefore we may conclude that the curves in region (i) are stable while those in region (iii) are unstable.

For the thick dashed curves in region (ii), a turning point exists at a maximum $\bar{M}$. In contrast to the corotating binary case, it is not fully established whether the stability of a solution sequence changes at this turning point or not. However, from the tendency of solution the sequences (i), (ii) and (iii), it is reasonable to expect that a part of the solutions on the left branches of the thick dashed curves in region (ii) are stable against gravitational collapse. From these results, it is reasonable to conclude that stable supramassive neutron stars can exist in irrotational binary systems as a result of tidal deformation, although the excess of the rest mass beyond the maximum rest mass of the spherical stars is tiny.

[1] C. Cutler et al., Phys. Rev. Lett. 70, 1984 (1993); K. S. Thorne, in Proceeding of Snowmass 95 Summer Study on Particle and Nuclear Astrophysics and Cosmology, edited by E. W. Kolb and R. Peccei (World Scientific, Singapore, 1995), p. 398.

[2] A. Abramovici et al. Science 256, 325 (1992) ; C. Bradaschia, et al. Nucl. Instrum. and Methods A289, 518 (1990) ; J. Hough, in Proceedings of the Sixth Marcel Grossmann Meeting, edited by H. Sato and T. Nakamura (World Scientific, Singapore, 1992), p.192 ; K. Kuroda et al., in Proceedings of the international conference on gravitational waves: Sources and Detectors, edited by I. Ciufolini and F. Fidecard(World Scientific, 1997), p.100.

[3] e.g., T. Piran, in Unsolved Problems in Astrophysics, edited by J. N. Bahcall and J. P. Ostriker (Princeton
University Press, 1997), 343.

[4] L.-X. Li and B. Paczynski, Astrophys. J. Lett. 507, L59 (1998).

[5] e.g., L. Blanchet, in Relativistic Gravitation and Gravitational Radiation edited by J.-P. Lasota and J.-A. Marck (Cambridge University Press, Cambridge, England, 1997), 33; Prog. Theor. Phys. Suppl. 136, 146 (1999):

C. M. Will, Prog. Theor. Phys. Suppl. 136, 158 (1999).

[6] X. Zhuge, J. M. Centrella, and S. L. W. McMillan, Phys. Rev. D 50, 6247 (1994); 54, 7261 (1996).

[7] Note however that if a dual recycling technique for a narrow band detector is available, it may be still possible to detect gravitational waves of high frequency beyond $\sim 1 \mathrm{kHz} \llbracket 1]$.

[8] K. Oohara and T. Nakamura, in Relativistic gravitation and gravitational radiation, edited by J.-P. Lasota and J.-A. Marck (Cambridge University Press, Cambridge, 199), 309; K. Oohara and T. Nakamura, Prog. Theor. Phys. Suppl. 136, 270 (1999).

[9] J. A. Font, M. Miller, W.-M. Suen, and M. Tobias, Phys. Rev. D 61, 044011 (2000); W.-M. Suen, Prog. Theor. Phys. Suppl. 136, 251 (1999).

[10] M. Shibata, Prog. Theor. Phys. 101, 251 (1999); M. Shibata, Prog. Theor. Phys. 101, 1199 (1999); M. Shibata, Phys. Rev. D 60, 104052 (1999).

[11] M. Shibata and K. Uryū, Phys. Rev. D 61, 064001 (2000).

[12] W. Landry and S. A. Teukolsky, preprint gr-qc/9912004 (1999).

[13] C. S. Kochanek, Astrophys. J. 398, 234 (1992); L. Bildsten and C. Cutler, Astrophys. J. 400, 175 (1992).

[14] S. Bonazzola, E. Gourgoulhon and J.-A. Marck, Phys. Rev. D 56; 7740 (1997); H. Asada, Phys. Rev. D 57, 7292 (1998).

[15] M. Shibata, Phys. Rev. D 58, 024012 (1998).

[16] S. A. Teukolsky, Astrophys. J. 504, 442 (1998).

[17] For example, H. Heiselberg and M. Hjorth-Jensen, nuclth/ 9902033.

[18] D. Lai, F. A. Rasio and S. L. Shapiro, Astrophys. J. Suppl. 88, 205 (1993); D. Lai, F. A. Rasio and S. L. Shapiro, Astrophys. J. 420, 811 (1994).

[19] J. C. Lombardi, Jr., F. A. Rasio and S. L. Shapiro, Phys. Rev. D 56, 3416 (1997).

[20] e.g., S. L. Shapiro and S. A. Teukolsky, Black Holes, White Dwarfs and Neutron Stars, (Wiley, New York, 1983).

[21] J. R. Wilson and G. J. Mathews, Phys. Rev. Lett. 75, 4161 (1995); J. R. Wilson, G. J. Mathews and P. Marronetti, Phys. Rev. D 54, 1317 (1996).

[22] S. Bonazzola, E. Gourgoulhon and J.-A. Marck, to be published in the proceedings of 19th Texas Symposium on Relativistic Astrophysics: Texas in Paris, Paris, France (1998); S. Bonazzola, E. Gourgoulhon and J.-A. Marck, Phys. Rev. Lett. 82, 892 (1999).

[23] P. Marronetti, G. J. Mathews and J. R. Wilson, to be published in the proceedings of 19th Texas Symposium on Relativistic Astrophysics: Texas in Paris, Paris, France (1998); P. Marronetti, G. J. Mathews and J. R. Wilson, Phys. Rev. D 60, 087301 (1999).

[24] K. Uryū and Y. Eriguchi, Phys. Rev. D. 61, 124023 
(2000).

[25] From a post Newtonian point of view, the solution in the conformal flatness approximation is expected to deviate from the correct solution. The magnitude of the deviation is e.g., of $\left.O[(v / c))^{4}\right]$ for $\Omega$ and $J$ and of $\left.O[(v / c))^{6}\right]$ for $M$ and $M_{0}$ where $v$ denotes characteristic magnitude of the orbital velocity: See, e.g., H. Asada and M. Shibata, Phys. Rev. D 54, 4944 (1996).

[26] F. Usui, K. Uryū and Y. Eriguchi, Phys. Rev. D 61, 024039 (2000).

[27] K. Uryū and Y. Eriguchi, Mon. Not. R. astr. Soc. 296, L1 (1998); Astrophys. J. Suppl. 118, 563 (1998); Mon. Not. R. astr. Soc. 303, 329 (1999); to be published in the proceedings of 19th Texas Symposium on Relativistic Astrophysics: Texas in Paris, Paris, France (1998).

[28] In the present computation, we typically took values of the type L mesh listed in Table II in 24. We also truncated the order of the Legendre expansion in Eq. (69) and Eq. (78) in 24] at $n_{\max }=32$ and $l_{\max }=10$ or 12 , typically, instead of infinity. We have checked the convergence of the numerical scheme and the effect of the truncation due to the Legendre expansion in [24. The above typical numbers for $n_{\max }$ and $l_{\max }$ have been shown to give accurate results.

[29] We changed $\widehat{d}$ by steps of 0.125 typically to construct a solution sequence and by steps of 0.0625 near to the minima of $\bar{J}$ and $\bar{M}$ when they appear on the sequence.

[30] Here, we refer to the general relativistic orbital instability as being that determined only by general relativistic gravity between two bodies and independent of any hydrodynamic effects and finite size effects, such as the orbital instability for a test particle orbiting near to a black hole. In this definition, the ISCO determined by the general relativistic instability should never depend on $(M / R)_{\infty}$ because of the strong equivalence principle.

[31] G. B. Cook, Phys. Rev. D 50, 5025 (1994).

[32] T. W. Baumgarte, Phys. Rev. D (2000), to be published (gr-qc/0004050).

[33] L. E. Kidder, C. M. Will, A. G. Wiseman, Class. Quantum Gravity 9, L125 (1992) ; Phys. Rev. D 47, 3281 (1993).

[34] T. Damour, B. R. Iyer, B. S. Sathyaprakash, Phys. Rev. D 57, 885 (1998).

[35] A. Buonanno, T. Damour, Phys. Rev. D 59, 084006 (1999).

[36] E. E. Flanagan, Phys. Rev. D 58, 124030 (1998): K. S. Thorne, Phys. Rev. D 58, 124031 (1998).

[37] According to the quadrupole formula for gravitational radiation reaction, $J_{\text {tot }}$ can decrease by $\sim 10 \%$, but the fraction of decrease of $M_{\mathrm{ADM}}$ will be at most $\sim 1 \%$ in one orbit around the innermost orbits. (In the terminology of the post Newtonian approximation, changes of $J_{\text {tot }}$ and $M_{\mathrm{ADM}}$ are proportional to $(v / c)^{5}$ and $(v / c)^{7}$, respectively, where $v$ is a characteristic speed of the orbital velocity.) Thus, $J_{\text {tot }} / M_{\mathrm{ADM}}^{2}$ is expected to decrease by $\sim 10 \%$.

[38] T. W. Baumgarte, S. L. Shapiro, and M. Shibata, Astrophys. J. 528, L29 (2000);

M. Shibata, T. W. Baumgarte and S. L. Shapiro, Astrophys. J. to be published (astro-ph/0005378.
[39] T. W. Baumgarte, G. B. Cook, M. A. Scheel, S. L. Shapiro and S. A. Teukolsky, Phys. Rev. D 57, 6181 (1998); ibid 57, 7299 (1998).

[40] The hydrodynamic interaction could be an important mechanism for outward angular momentum transportation in particular for the case when spiral arms are formed in the outer region of a merger object and an ellipsoidal object are formed in the inner region. However, recent results of numerical simulations for the merger of irrotational binaries [11] show that such structures are not formed significantly, which indicates that the timescale of the transportation could not be short enough to increase the specific angular momentum of a certain fraction of fluid elements.

[41] Note that $j$ at the ISCO for a test particle orbiting progradely in the equatorial plane of a Kerr black hole with $q=0.9$ is $j \approx 2.1 M_{\mathrm{BH}}[20$. (For the maximally rotating Kerr black hole $(q=1), j$ at the ISCO is $M_{\mathrm{BH}}$.)

[42] e.g., K. Oohara, T. Nakamura and M. Shibata, Prog. Theor. Phys. Suppl. 128, 183 (1997).

[43] R. Sorkin, Astrophys. J. 249, 254 (1981); J. R. Ipser and G. Horowitz, Astrophys. J. 232, 863 (1979); J. Katz, Mon. Not. R. astr. Soc. 183, 765 (1978). 


\begin{tabular}{|c|c|c|c|c|c|c|c|}
\hline$(M / R)_{\infty}$ & $\widehat{d}$ & $\bar{\rho}_{\max }$ & $\bar{M}_{0}$ & $\bar{M}$ & $M_{0} \Omega$ & $J_{\text {tot }} / M_{\mathrm{ADM}}^{2}$ & $d_{G} / M$ \\
\hline \multicolumn{8}{|c|}{$n=0.5$} \\
\hline 0.10 & 1.1250 & $2.50 \mathrm{E}-01$ & $4.41 \mathrm{E}-02$ & $4.12 \mathrm{E}-02$ & $1.07 \mathrm{E}-02$ & $1.12 \mathrm{E}+00$ & $1.28 \mathrm{E}+01$ \\
\hline 0.12 & 1.1250 & $2.83 \mathrm{E}-01$ & $5.52 \mathrm{E}-02$ & $5.08 \mathrm{E}-02$ & $1.46 \mathrm{E}-02$ & $1.05 \mathrm{E}+00$ & $1.03 \mathrm{E}+01$ \\
\hline 0.14 & 1.1250 & $3.16 \mathrm{E}-01$ & $6.65 \mathrm{E}-02$ & $6.04 \mathrm{E}-02$ & $1.92 \mathrm{E}-02$ & $9.95 \mathrm{E}-01$ & $8.56 \mathrm{E}+00$ \\
\hline 0.17 & 1.1250 & 3.69E-01 & $8.40 \mathrm{E}-02$ & $7.45 \mathrm{E}-02$ & 2.72E-02 & $9.41 \mathrm{E}-01$ & $6.67 \mathrm{E}+00$ \\
\hline 0.19 & 1.1250 & $4.08 \mathrm{E}-01$ & $9.56 \mathrm{E}-02$ & $8.36 \mathrm{E}-02$ & 3.35E-02 & $9.15 \mathrm{E}-01$ & $5.74 \mathrm{E}+00$ \\
\hline \multicolumn{8}{|c|}{$n=0.66667$} \\
\hline 0.10 & 1.1875 & $1.60 \mathrm{E}-01$ & $6.06 \mathrm{E}-02$ & $5.68 \mathrm{E}-02$ & $1.06 \mathrm{E}-02$ & $1.10 \mathrm{E}+00$ & $1.28 \mathrm{E}+01$ \\
\hline 0.12 & 1.1875 & $1.90 \mathrm{E}-01$ & 7.41E-02 & $6.84 \mathrm{E}-02$ & $1.45 \mathrm{E}-02$ & $1.03 \mathrm{E}+00$ & $1.03 \mathrm{E}+01$ \\
\hline 0.14 & 1.1875 & $2.21 \mathrm{E}-01$ & $8.74 \mathrm{E}-02$ & 7.97E-02 & $1.89 \mathrm{E}-02$ & $9.81 \mathrm{E}-01$ & $8.56 \mathrm{E}+00$ \\
\hline 0.17 & 1.1875 & $2.75 \mathrm{E}-01$ & $1.07 \mathrm{E}-01$ & $9.55 \mathrm{E}-02$ & $2.68 \mathrm{E}-02$ & $9.28 \mathrm{E}-01$ & $6.69 \mathrm{E}+00$ \\
\hline 0.19 & 1.1875 & $3.16 \mathrm{E}-01$ & $1.19 \mathrm{E}-01$ & $1.05 \mathrm{E}-01$ & $3.30 \mathrm{E}-02$ & $9.03 \mathrm{E}-01$ & $5.73 \mathrm{E}+00$ \\
\hline \multicolumn{8}{|c|}{$n=0.8$} \\
\hline 0.10 & 1.1875 & $1.13 \mathrm{E}-01$ & 7.77E-02 & 7.30E-02 & $1.07 \mathrm{E}-02$ & $1.10 \mathrm{E}+00$ & $1.27 \mathrm{E}+01$ \\
\hline 0.12 & 1.1875 & $1.39 \mathrm{E}-01$ & $9.32 \mathrm{E}-02$ & $8.64 \mathrm{E}-02$ & $1.45 \mathrm{E}-02$ & $1.03 \mathrm{E}+00$ & $1.03 \mathrm{E}+01$ \\
\hline 0.14 & 1.1875 & $1.69 \mathrm{E}-01$ & $1.08 \mathrm{E}-01$ & $9.88 \mathrm{E}-02$ & $1.89 \mathrm{E}-02$ & $9.75 \mathrm{E}-01$ & $8.52 \mathrm{E}+00$ \\
\hline 0.17 & 1.1875 & $2.21 \mathrm{E}-01$ & $1.28 \mathrm{E}-01$ & $1.15 \mathrm{E}-01$ & $2.68 \mathrm{E}-02$ & $9.22 \mathrm{E}-01$ & $6.63 \mathrm{E}+00$ \\
\hline 0.19 & 1.1875 & $2.63 \mathrm{E}-01$ & $1.41 \mathrm{E}-01$ & $1.25 \mathrm{E}-01$ & $3.28 \mathrm{E}-02$ & $8.97 \mathrm{E}-01$ & $5.71 \mathrm{E}+00$ \\
\hline \multicolumn{8}{|c|}{$n=1.0$} \\
\hline 0.10 & 1.2500 & $6.96 \mathrm{E}-02$ & $1.12 \mathrm{E}-01$ & $1.05 \mathrm{E}-01$ & $1.06 \mathrm{E}-02$ & $1.09 \mathrm{E}+00$ & $1.27 \mathrm{E}+01$ \\
\hline 0.12 & 1.2500 & $9.12 \mathrm{E}-02$ & $1.30 \mathrm{E}-01$ & $1.21 \mathrm{E}-01$ & $1.44 \mathrm{E}-02$ & $1.02 \mathrm{E}+00$ & $1.03 \mathrm{E}+01$ \\
\hline 0.14 & 1.2500 & $1.17 \mathrm{E}-01$ & $1.46 \mathrm{E}-01$ & 1.35E-01 & $1.87 \mathrm{E}-02$ & $9.71 \mathrm{E}-01$ & $8.52 \mathrm{E}+00$ \\
\hline 0.17 & 1.2500 & 1.69E-01 & $1.66 \mathrm{E}-01$ & $1.50 \mathrm{E}-01$ & 2.63E-02 & $9.19 \mathrm{E}-01$ & $6.66 \mathrm{E}+00$ \\
\hline 0.19 & 1.2500 & $2.15 \mathrm{E}-01$ & $1.75 \mathrm{E}-01$ & $1.57 \mathrm{E}-01$ & $3.20 \mathrm{E}-02$ & 8.95E-01 & $5.75 \mathrm{E}+00$ \\
\hline \multicolumn{8}{|c|}{$n=1.25$} \\
\hline 0.10 & 1.2500 & $3.96 \mathrm{E}-02$ & $1.73 \mathrm{E}-01$ & $1.64 \mathrm{E}-01$ & $1.06 \mathrm{E}-02$ & $1.08 \mathrm{E}+00$ & $1.26 \mathrm{E}+01$ \\
\hline 0.12 & 1.2500 & $5.65 \mathrm{E}-02$ & $1.92 \mathrm{E}-01$ & $1.80 \mathrm{E}-01$ & $1.43 \mathrm{E}-02$ & $1.02 \mathrm{E}+00$ & $1.02 \mathrm{E}+01$ \\
\hline 0.14 & 1.2500 & 7.93E-02 & 2.07E-01 & $1.92 \mathrm{E}-01$ & $1.85 \mathrm{E}-02$ & $9.67 \mathrm{E}-01$ & $8.50 \mathrm{E}+00$ \\
\hline 0.17 & 1.2500 & $1.19 \mathrm{E}-01$ & $2.17 \mathrm{E}-01$ & $2.00 \mathrm{E}-01$ & $2.40 \mathrm{E}-02$ & $9.25 \mathrm{E}-01$ & $7.02 \mathrm{E}+00$ \\
\hline
\end{tabular}

TABLE I. Characteristic quantities for irrotational binary neutron stars for configurations at $\bar{d}_{G}=\bar{d}_{R}$. Here, $\bar{\rho}_{\text {max }}$ is the maximum rest mass density. Note that for spherical stars, the maximum value of the normalized rest mass and the compactness at the maximum $\left(\bar{M}_{0},(M / R)_{\infty}\right)$ are $(0.152,0.316)$ for $n=0.5,(0.155,0.278)$ for $\mathrm{n}=0.66667,(0.162,0.252)$ for $n=0.8,(0.180$, $0.214)$ for $\mathrm{n}=1$, and $(0.217,0.172)$ for $\mathrm{n}=1.25$. Because of an uncertainty for determination of the configuration with cusps, $M_{0} \Omega$ and $M_{\mathrm{ADM}} \Omega$ have an error of $1-2 \%$ for each $(M / R)_{\infty}$. 


\begin{tabular}{|c|c|c|c|c|c|c|c|c|}
\hline$(M / R)_{\infty}$ & $\widehat{d}$ & $\bar{\rho}_{\max }$ & $\bar{M}_{0}$ & $\bar{M}$ & $M_{0} \Omega$ & $M_{\mathrm{ADM}} \Omega$ & $J_{\text {tot }} / M_{\mathrm{ADM}}^{2}$ & $d_{G} / M$ \\
\hline 0.03 & 1.1875 & 0.124 & $9.89 \mathrm{E}-03$ & $9.69 \mathrm{E}-03$ & $1.55 \mathrm{E}-03$ & $3.04 \mathrm{E}-03$ & 1.88 & 47.7 \\
\hline 0.06 & 1.1875 & 0.183 & $2.34 \mathrm{E}-02$ & $2.25 \mathrm{E}-02$ & $4.59 \mathrm{E}-03$ & 8.83E-03 & 1.38 & 22.9 \\
\hline 0.10 & 1.2500 & 0.251 & $4.41 \mathrm{E}-02$ & $4.12 \mathrm{E}-02$ & $1.04 \mathrm{E}-02$ & $1.94 \mathrm{E}-02$ & 1.12 & 13.1 \\
\hline 0.12 & 1.2500 & 0.284 & $5.52 \mathrm{E}-02$ & $5.08 \mathrm{E}-02$ & $1.41 \mathrm{E}-02$ & $2.60 \mathrm{E}-02$ & 1.05 & 10.6 \\
\hline 0.14 & 1.2500 & 0.317 & $6.65 \mathrm{E}-02$ & $6.04 \mathrm{E}-02$ & $1.85 \mathrm{E}-02$ & $3.36 \mathrm{E}-02$ & 0.994 & 8.76 \\
\hline 0.17 & 1.3125 & 0.371 & $8.40 \mathrm{E}-02$ & 7.45E-02 & $2.55 \mathrm{E}-02$ & $4.53 \mathrm{E}-02$ & 0.938 & 6.97 \\
\hline 0.19 & 1.3125 & 0.410 & $9.56 \mathrm{E}-02$ & $8.36 \mathrm{E}-02$ & $3.14 \mathrm{E}-02$ & $5.50 \mathrm{E}-02$ & 0.912 & 6.00 \\
\hline 0.21 & 1.3125 & 0.457 & $1.07 \mathrm{E}-01$ & $9.22 \mathrm{E}-02$ & $3.84 \mathrm{E}-02$ & $6.60 \mathrm{E}-02$ & 0.899 & 5.20 \\
\hline 0.24 & 1.3750 & 0.529 & $1.24 \mathrm{E}-01$ & $1.04 \mathrm{E}-01$ & $4.83 \mathrm{E}-02$ & $8.13 \mathrm{E}-02$ & 0.872 & 4.37 \\
\hline 0.27 & 1.5000 & 0.628 & $1.39 \mathrm{E}-01$ & $1.14 \mathrm{E}-01$ & $5.79 \mathrm{E}-02$ & $9.54 \mathrm{E}-02$ & 0.858 & 3.81 \\
\hline 0.30 & 1.6250 & 0.773 & $1.49 \mathrm{E}-01$ & $1.21 \mathrm{E}-01$ & $6.79 \mathrm{E}-02$ & 0.110 & 0.851 & 3.37 \\
\hline
\end{tabular}

TABLE II. The same as Table I but for a configuration with $n=0.5$ at $\bar{d}_{G}=\bar{d}_{\mathrm{dyn}}$. We include $M_{\mathrm{ADM}} \Omega$ in this Table. Because of an uncertainty for determining the minima of $J_{\text {tot }}$ and $M_{\mathrm{ADM}}, M_{0} \Omega$ and $M_{\mathrm{ADM}} \Omega$ have an error of $1-2 \%$ for each $(M / R)_{\infty}$

\begin{tabular}{ccccccccc}
\hline \hline$(M / R)_{\infty}$ & $\widehat{d}$ & $\bar{\rho}_{\max }$ & $\bar{M}_{0}$ & $\bar{M}$ & $M_{0} \Omega$ & $M_{\mathrm{ADM}} \Omega$ & $J_{\text {tot }} / M_{\mathrm{ADM}}^{2}$ & $d_{G} / M$ \\
\hline 0.17 & 1.1875 & $2.75 \mathrm{E}-01$ & $1.07 \mathrm{E}-01$ & $9.55 \mathrm{E}-02$ & $2.68 \mathrm{E}-02$ & $4.78 \mathrm{E}-02$ & 0.928 \\
0.19 & 1.1875 & $3.16 \mathrm{E}-01$ & $1.19 \mathrm{E}-01$ & $1.05 \mathrm{E}-01$ & $3.30 \mathrm{E}-02$ & $5.82 \mathrm{E}-02$ & 0.903 \\
0.21 & 1.2500 & $3.64 \mathrm{E}-01$ & $1.31 \mathrm{E}-01$ & $1.14 \mathrm{E}-01$ & $3.91 \mathrm{E}-02$ & $6.79 \mathrm{E}-02$ & 0.884 & 5.73 \\
0.24 & 1.3125 & $4.56 \mathrm{E}-01$ & $1.45 \mathrm{E}-01$ & $1.24 \mathrm{E}-01$ & $4.93 \mathrm{E}-02$ & $8.42 \mathrm{E}-02$ & 0.865 & 4.24 \\
0.27 & 1.4375 & $5.85 \mathrm{E}-01$ & $1.54 \mathrm{E}-01$ & $1.30 \mathrm{E}-01$ & $5.78 \mathrm{E}-02$ & $9.75 \mathrm{E}-02$ & 0.855 & 3.74 \\
\hline \hline
\end{tabular}

TABLE III. The same as Table II but for a configuration with $n=0.66667$ at $\bar{d}_{G}=\bar{d}_{\text {dyn. }}$. Because of an uncertainty for determining the minima of $J_{\text {tot }}$ and $M_{\mathrm{ADM}}, M_{0} \Omega$ has an error of $1-2 \%$ for each $(M / R)_{\infty}$. Note that $\bar{d}_{\mathrm{dyn}}$ is nearly equal to $\bar{d}_{R}$ for $(M / R)_{\infty} \leq 0.19$ but not for $(M / R)_{\infty}>0.2$. 


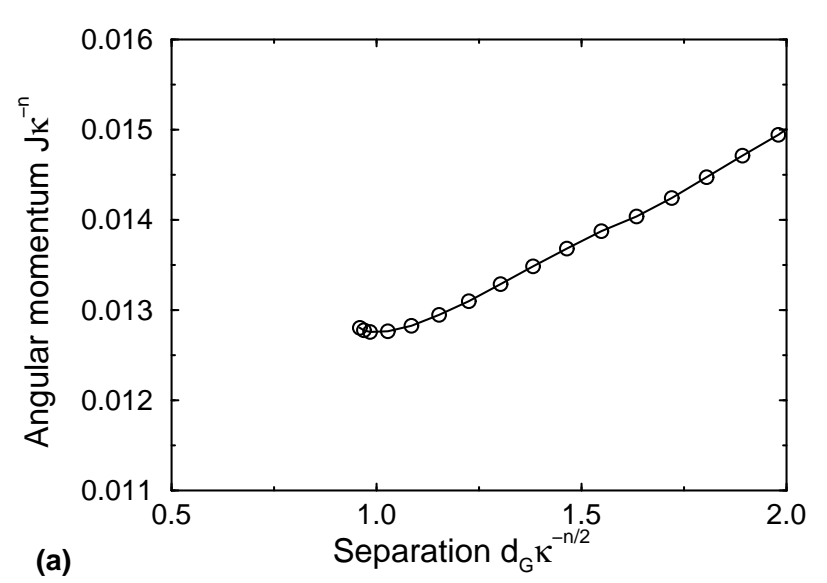

\begin{tabular}{cc}
\hline \hline$(M / R)_{\infty}$ & $(a, b, c)$ \\
\hline $0.03-0.17$ & $(0.2849,0.422,0.29)$ \\
$0.03-0.19$ & $(0.2878,0.392,0.93)$ \\
\hline$(M / R)_{\infty}$ & $\left(a^{\prime}, b^{\prime}, c^{\prime}\right)$ \\
\hline $0.03-0.17$ & $(0.2849,0.245,-0.08)$ \\
$0.03-0.19$ & $(0.2864,0.200,0.18)$ \\
\hline \hline
\end{tabular}

TABLE IV. Coefficients of the fitting formula Eq. (20) for the results for $n=0.5$ (Table II). The column headed $(M / R)_{\infty}$ shows the data sets used for each fitting.
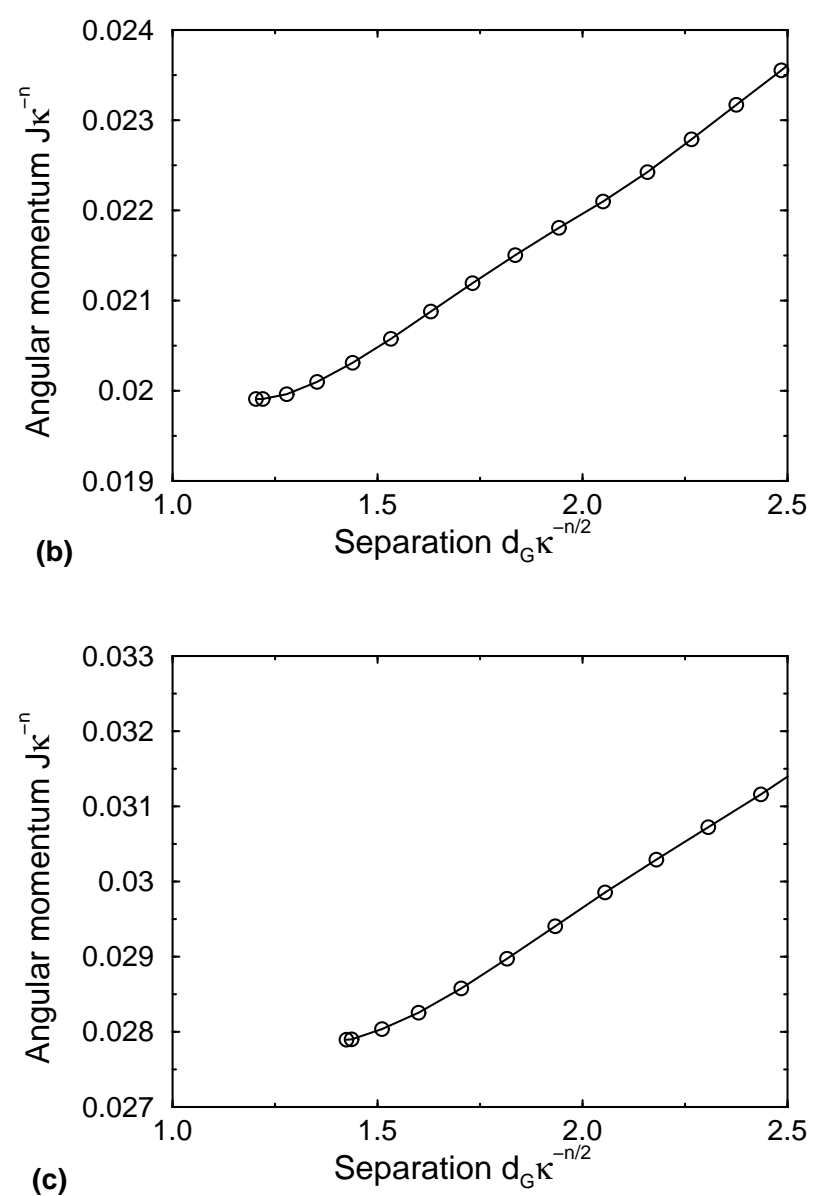

FIG. 1. $\quad \bar{J}$ as a function of $\bar{d}_{G}$ for (a) $n=0.5$, (b) $n=0.66667$ and (c) $n=0.8$ and for $(M / R)_{\infty}=0.19$. The points of smallest $\bar{d}_{G}$ along the curves correspond to $\bar{d}_{G}=\bar{d}_{R}$ where neutron stars have cusps at the inner edges of the stellar surfaces. 
(a) $n>n_{\mathrm{cr}}$

(b) $\mathrm{n}<\mathrm{n}_{\mathrm{cr}}$

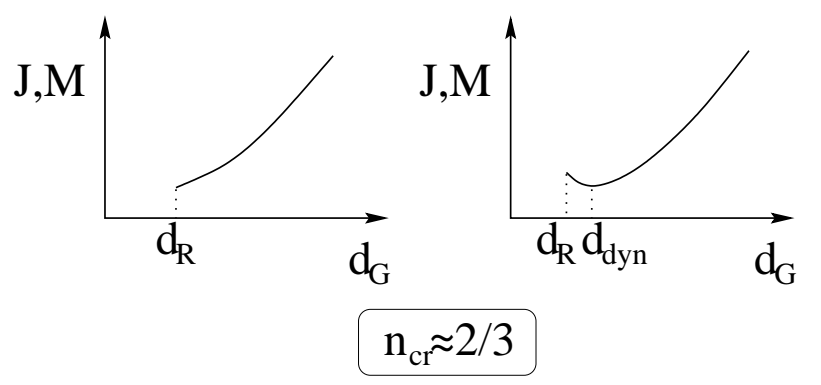

FIG. 2. Schematic figure of $J$ and $M$ as functions of $d_{G}$ for sequences of constant rest mass. $d_{R}$ corresponds to the point where neutron stars have cusps at the inner edges of the stellar surfaces. $d_{\mathrm{dyn}}$ corresponds to a turning point along the sequence.

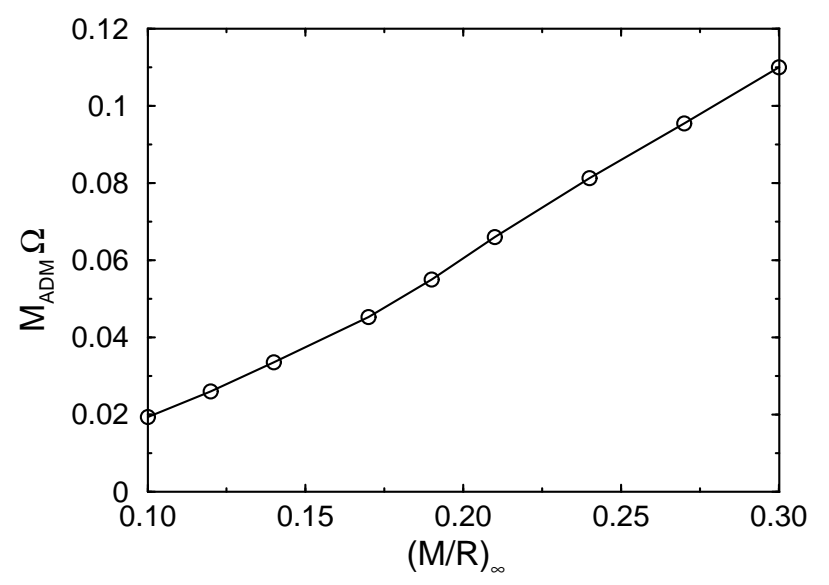

FIG. 3. Plot of $(M / R)_{\infty}-\Omega M_{\mathrm{ADM}}$ relations at the ISCO $\left(d_{G} \approx d_{\text {dyn }}\right)$ for binary neutron stars with $n=0.5$ and $n=0.66667$.

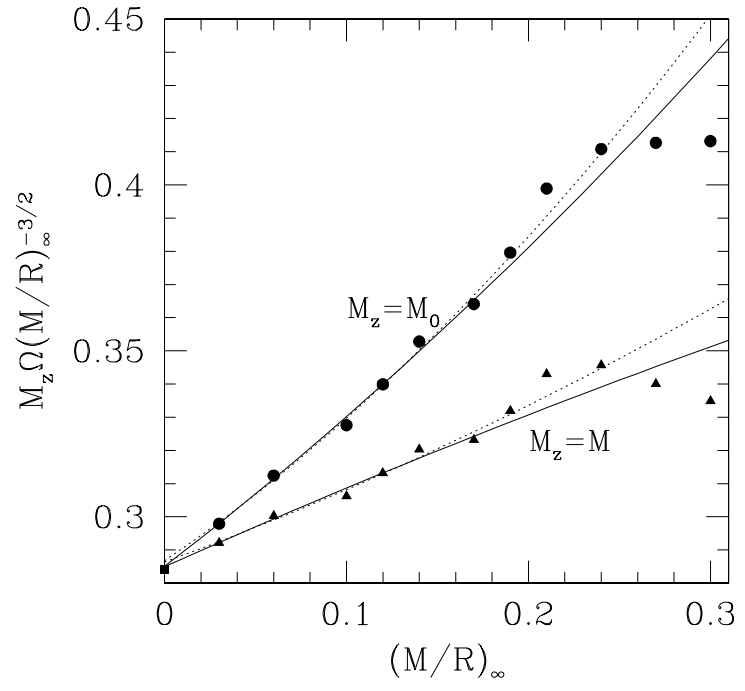

FIG. 4. $M_{0} \Omega$ (filled circles) and $M \Omega$ (filled triangles) at the ISCO $\left(d_{G} \simeq d_{\mathrm{dyn}}\right)$ as a function of $(M / R)_{\infty}$ for $n=0.5$. The solid and dotted lines denote the fitting formulae derived using the data sets with $0.03 \leq(M / R)_{\infty} \leq 0.17$ (solid lines) and $0.03 \leq(M / R)_{\infty} \leq 0.19$ (dotted lines), respectively. The squares at $(M / R)_{\infty}=0.0$ denote the results from the Newtonian computation.

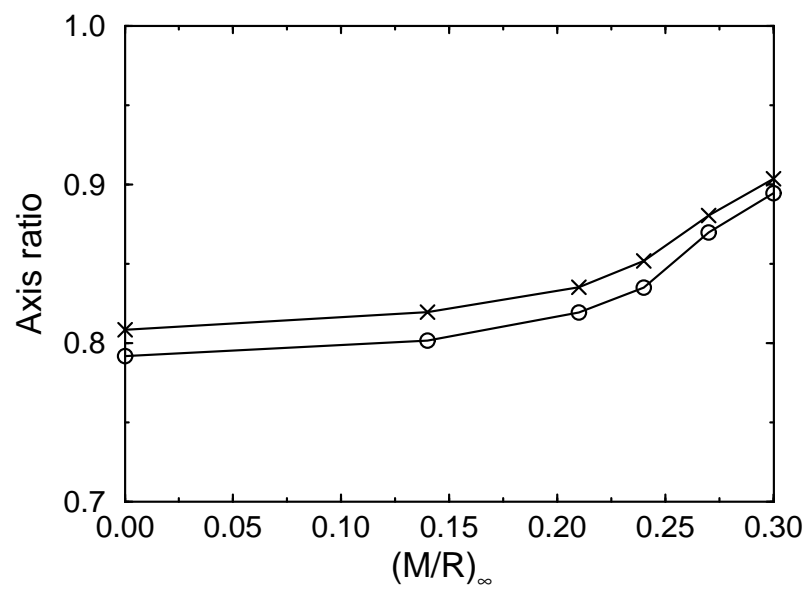

FIG. 5. The ratios $a_{2} / a_{1}$ (circles) and $a_{3} / a_{1}$ (crosses) as functions of $(M / R)_{\infty}$ at ISCOs for $n=0.5$. Values at $(M / R)_{\infty}=0.0$ are those from the Newtonian computation. 

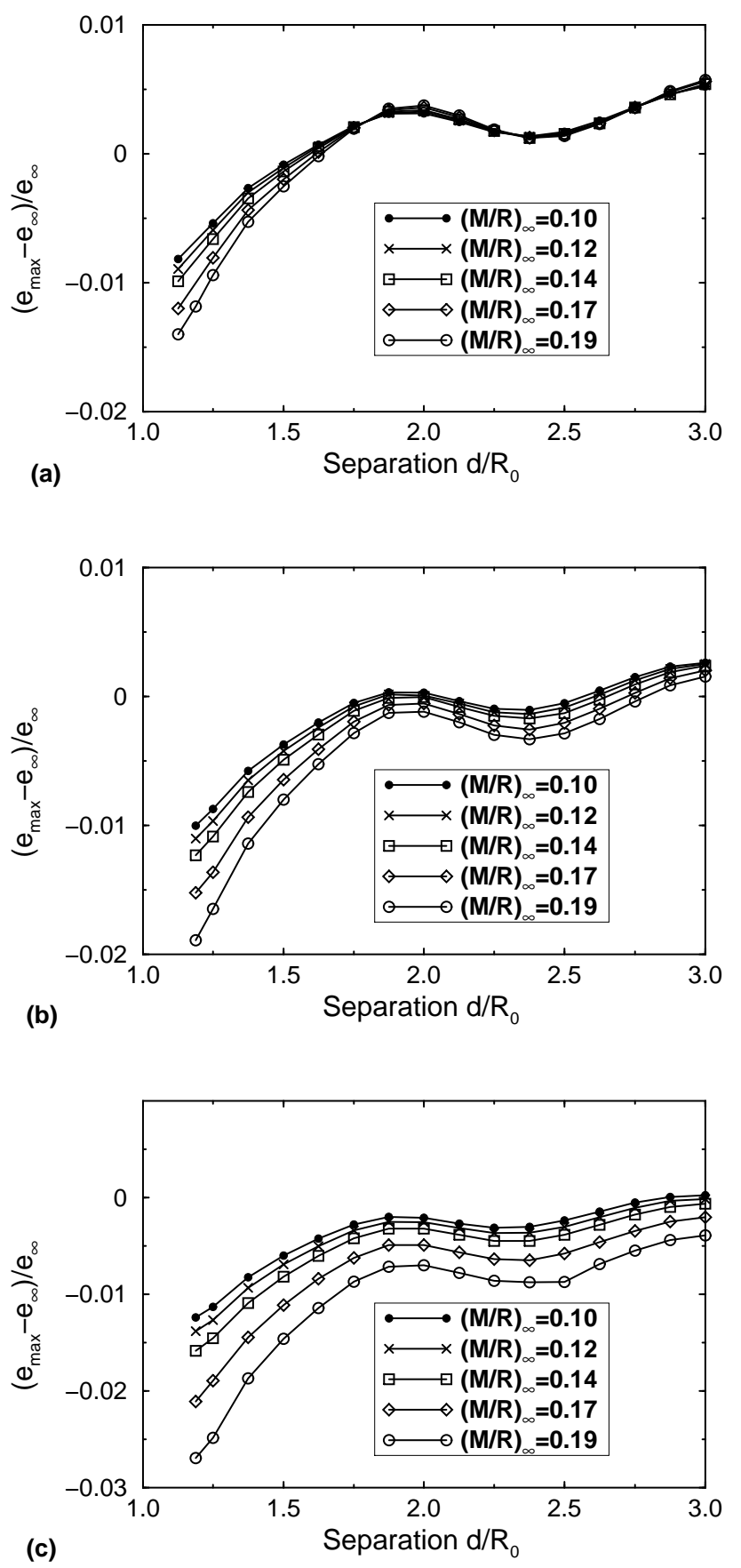

FIG. 6. $\quad\left(e_{\max }-e_{\infty}\right) / e_{\infty}$ as a function of $\widehat{d}$ for (a) $n=0.5$, (b) $n=0.66667$ and (c) $n=0.8$. Cusps appear at the inner edges of the stellar surfaces at the point of smallest separation on each curve.

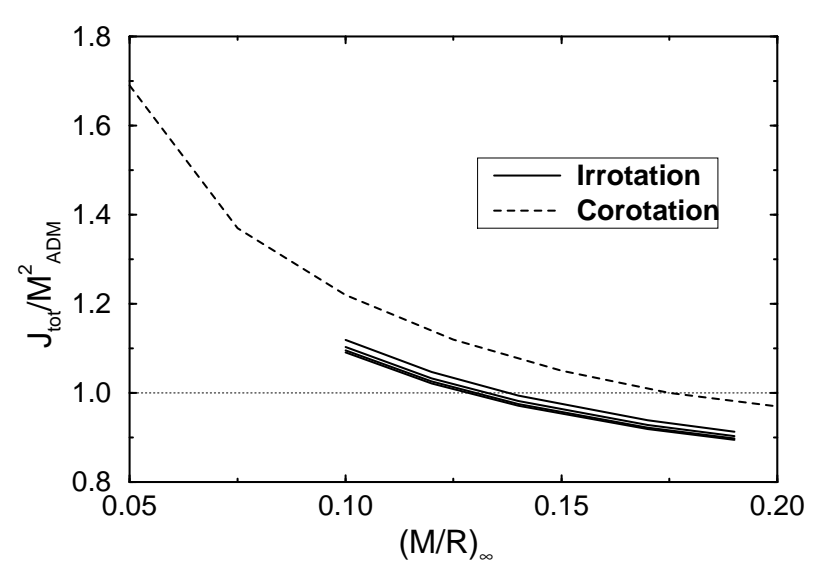

FIG. 7. Relation between $(M / R)_{\infty}$ and $J_{\text {tot }} / M_{\mathrm{ADM}}^{2}$ at the innermost orbits of binary neutron stars. The solid lines denote the relation for irrotational binaries with $n=0.5$, 0.66667, 0.8 and 1 from top to bottom, and the dashed line is for corotating binaries with $n=1$. For the irrotational case, the models at $\bar{d}_{G}=\bar{d}_{R}$ are chosen as the innermost orbits, and for the corotating case, the models at the energy and angular momentum minima are chosen. 

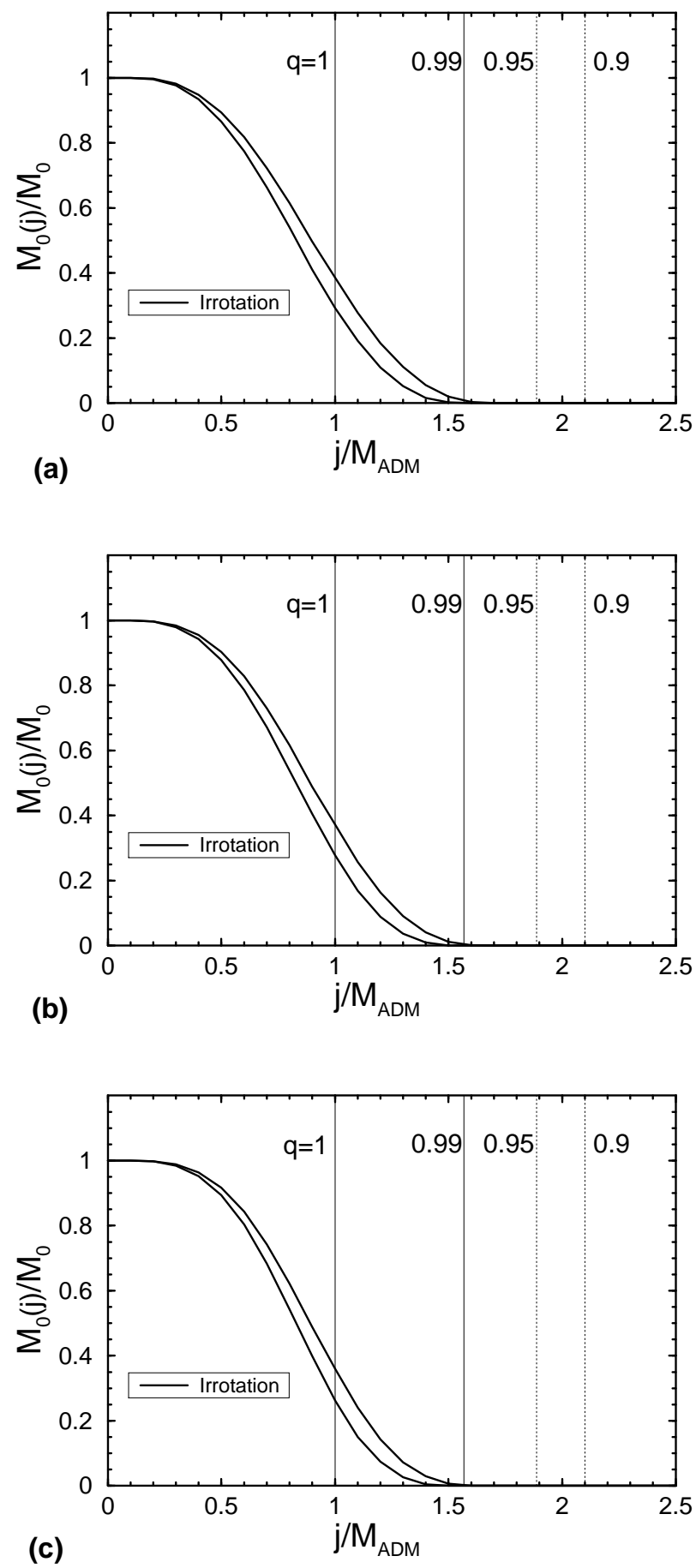
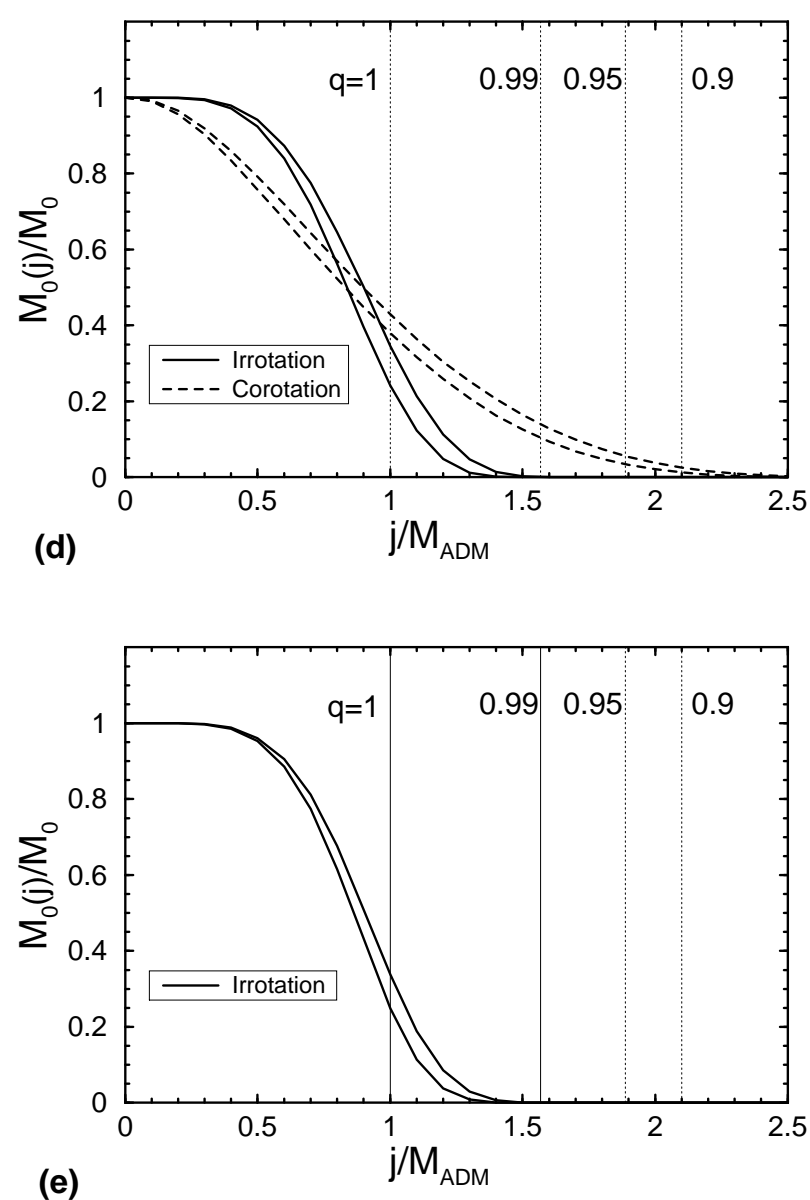

FIG. 8. Fraction of rest mass with specific angular momentum larger than $j$ for the innermost orbits for $(M / R)_{\infty}=0.14$ and 0.17 and for (a) $n=0.5$, (b) $n=0.66667$, (c) $n=0.8$, (d) $n=1$, and (e) $n=1.25$. The solid and dashed lines correspond to the results for irrotational and corotating binaries, respectively. The lines for $(M / R)_{\infty}=0.17$ appear as upper lines in each panel. For the irrotational case, the models at $\bar{d}_{G}=\bar{d}_{\text {dyn }}$ are chosen for $n=0.5$, and the models at $\bar{d}_{G}=\bar{d}_{R}$ are chosen for the other $n$. For the corotating case, the models for which the two surfaces come into contact are chosen. 

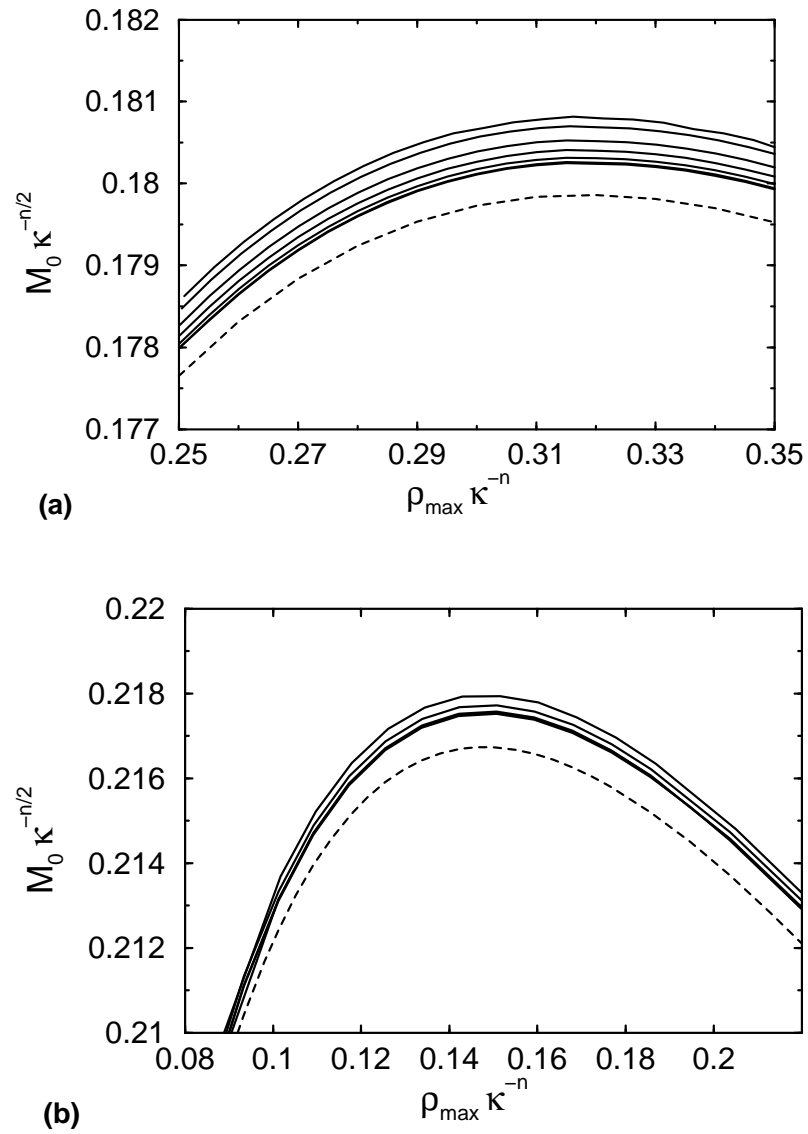

FIG. 9. $\quad \bar{M}_{0}$ as a function of $\bar{\rho}_{\max }$ (a) Solid lines from top to bottom correspond to $\widehat{d}=1.3125,1.375,1.5,1.625,1,75$, 1,875 and 2 for the $n=1$ case. (b) Solid lines from top to bottom correspond to $\widehat{d}=1.3125,1.5,1.75,2$ and 2.5 for the $n=1.25$ case. A dashed line corresponds to a solution of the TOV equations in each panel.
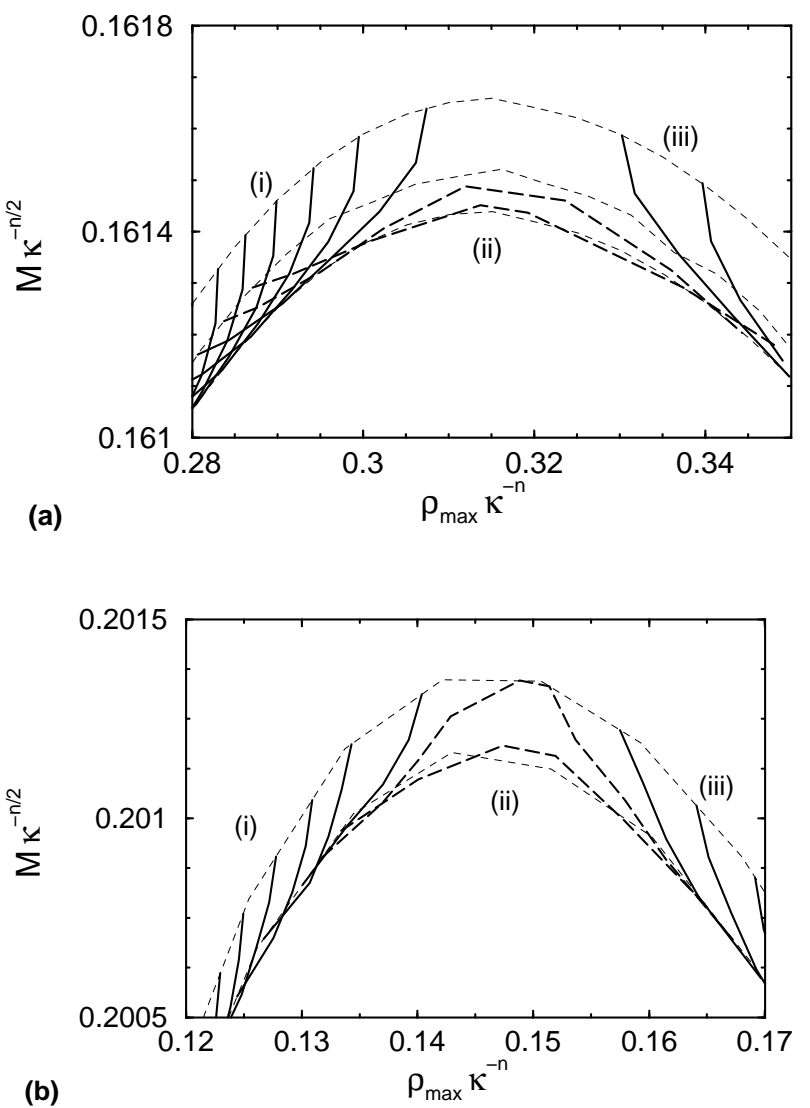

FIG. 10. Sequences for constant $\bar{M}_{0}$ are plotted in the $\bar{\rho}_{\max }-\bar{M}$ plane (thick solid lines and thick long dashed lines). (a) $n=1$ case. From left to right, the $\bar{M}_{0}$ of each curve changes from 0.1797 to 0.1802 in steps of 0.0001 (solid lines) in region (i) (i.e., $\bar{\rho}_{\max }<0.31$ ). In region (ii), two long dashed lines with $\bar{M}_{0}=0.1803$ and 0.1804 (upper one and lower one respectively) are plotted. In region (iii) (i.e., $\bar{\rho}_{\max }>0.31$ ), $\bar{M}_{0}$ is taken from 0.1802 to 0.1801 in steps of 0.0001 (solid lines). The upper, middle and lower thin dashed curves correspond to the relation between $\bar{M}$ and $\bar{\rho}_{\max }$ for $\widehat{d}=2.0,1.3125$ and 1.5 , respectively. (b) $n=1.25$ case. From left to right, the $\bar{M}_{0}$ of each curve changes from 0.2164 to 0.2174 in steps of 0.0002 (solid lines) in region (i) (i.e., $\bar{\rho}_{\max }<0.14$ ). In region (ii), two long dashed lines with $\bar{M}_{0}=0.2175$ and 0.2176 (upper one and lower one respectively) are plotted. In region (iii) (i.e., $\bar{\rho}_{\max }>0.14$ ), $\bar{M}_{0}$ is taken from 0.2174 to 0.2170 in steps of 0.0002 (solid lines). The upper and lower thin dashed curves correspond to the relations between $\bar{M}$ and $\bar{\rho}_{\max }$ for $\widehat{d}=1.875$ and 1.3125 , respectively. 